\title{
Review and Comparison of Computational Approaches for Joint Longitudinal and Time-to-Event Models
}

\author{
Allison K.C. Furgal ${ }^{1}{ }^{\oplus}$, Ananda Sen $^{1,2}$ and Jeremy M.G. \\ Taylor $^{1}$
}

${ }^{1}$ Biostatistics Department, School of Public Health, University of Michigan, 1415 Washington Heights, Ann Arbor, MI, 48109, USA

${ }^{2}$ Department of Family Medicine, Michigan Medicine, University of Michigan, 1018 Fuller St, Ann Arbor, MI 48104, USA

E-mail: acullen@umich.edu

\section{Summary}

Joint models for longitudinal and time-to-event data are useful in situations where an association exists between a longitudinal marker and an event time. These models are typically complicated due to the presence of shared random effects and multiple submodels. As a consequence, software implementation is warranted that is not prohibitively time consuming. While methodological research in this area continues, several statistical software procedures exist to assist in the fitting of some joint models. We review the available implementation for frequentist and Bayesian models in the statistical programming languages R, SAS and Stata. A description of each procedure is given including estimation techniques, input and data requirements, available options for customisation and some available extensions, such as competing risks models. The software implementations are compared and contrasted through extensive simulation, highlighting their strengths and weaknesses. Data from an ongoing trial on adrenal cancer patients are used to study different nuances of software fitting on a practical example.

Key words: computational approaches; joint model; longitudinal data; software comparison; survival data; time-to-event data.

\section{Introduction}

Studies often measure both a longitudinal process and an associated event time. A case in point is a study of time to AIDS diagnosis and CD4 counts (Huang et al., 2011; Wang \& Taylor, 2001; Wulfsohn \& Tsiatis, 1997). In cancer, increasing PSA levels are known to be associated with prostate cancer recurrence (Proust-Lima \& Taylor, 2009). Other examples include study of biomarkers in cancer vaccine trials, quality of life measurements in cancer, time-tofailure and degradation in engineering applications (Brown \& Ibrahim, 2003; Ibrahim et al., 2010; Lehmann, 2009). In such contexts, separate models for the longitudinal and survival components that do not take into account the dependence between the longitudinal and survival processes produce inefficient results and are prone to bias. Using the longitudinal process as a time-dependent covariate in the survival model requires the unrealistic assumptions that 
the longitudinal measurement has negligible measurement error and that there are observations at every event time. Traditional two-stage models account for the measurement error in the survival model by fitting the longitudinal process first and subsequently using the estimated trajectory as a covariate in the survival model (Tsiatis et al., 1995). However, this strategy fails to account for the possible informative censoring in the longitudinal process (Mccrink et al., 2013). A fully specified joint model incorporates the dependence between the longitudinal and survival components properly and provides efficient inference that is less prone to bias.

Apart from the scenario when the interrelated longitudinal and survival processes are both of interest, joint models are also useful when the event time causes non-informative censoring on the observed longitudinal process that may be of primary interest (Rizopoulos, 2012, pp. $51,90)$. While there are differing approaches to formulate joint models, in this review, we shall focus on random effects models as the software platform is developed almost exclusively in this framework.

Early work on joint models dates back to the mid-nineties (De Gruttola \& Tu, 1994). Since then, a body of work has steadily emerged in this area of research (Henderson et al., 2000; Wang \& Taylor, 2001; Wulfsohn \& Tsiatis, 1997). Some early Bayesian work on this topic includes those by Faucett and Thomas (1996), and Brown and Ibrahim (2003). More recently, there have been several extensions in the directions of dynamic predictions and prognostic tools (Proust-Lima \& Taylor, 2009; Taylor et al., 2013; Rizopoulos et al., 2014), competing risks (Elashoff et al., 2007; Hu et al., 2009), recurrent events (Liu \& Huang, 2009), multiple longitudinal variables (Li et al., 2007), cure rates (Yu et al., 2004) and diagnostics (Huang et al., 2009). A comprehensive review of early work on models and methods related to joint modelling of longitudinal and survival processes has appeared in Tsiatis and Davidian (2004).

With multiple submodels and shared random effects, joint models can become complicated and difficult to utilise. This motivates the need for creating an efficient computational platform to fit these models. Due to the complexity of joint models, implementation can be slow but can still be useful for those wanting to fit relatively simple joint models for data analysis. Major statistical softwares such as R, SAS and Stata include joint modelling functions. The purpose of this article is to provide a comprehensive review of associated implementation issues and explore new applications in related software platforms. There has been limited review of software implementation in the joint modelling literature. Some early reviews listed softwares available at the time (Gould et al., 2014; Mccrink et al., 2013), utilised a single software for data analysis (Rizopoulos, 2012) or compared a couple in the context of analysing a dataset (Mccrink et al., 2013). Other reviews have compared selected softwares, such as WinBUGS and SAS PROC NLMIXED (Guo \& Carlin, 2004). Documentation of the $\%$ JM macro in SAS compares the available features of the macro to the JM package in R (Garcia-Hernandez \& Rizopoulos, 2015). A recent review of joint modelling literature by Sudell et al. (2016) discussed the frequency with which each joint modelling software was used. More recently, Yuen and Mackinnon (2016) compare some of the available software with an application to a dataset documenting time to psychosis transition.

There are several ways in which our review differs from those in the existing literature. Most of the reviews focus primarily on the survival component of the joint analysis. By contrast, we provide findings from simulation that assess simultaneous performance of the time-to-event and the longitudinal model. Our documentation is also more comprehensive than most reviews covering specialised topics such as latent classes, competing risks, multiple longitudinal outcomes and more. Through extensive simulation, we present a comprehensive appraisal of the different implementations. In addition, the different softwares are contrasted by means of their 
performance when used to fit to data from an ongoing trial on adrenal cancer patients enrolled in the University of Michigan Comprehensive Cancer Center.

In Section 2, we will review the joint modelling framework and associated estimation methods along with the computational algorithms. The next section demonstrates the software capabilities with an example data analysis. We will discuss software implementation in Section 4. This includes the JM and joineR packages in R, the $\%$ JM macro in SAS and the st jm command in Stata. In Section 5, we describe our simulations. Section 6 outlines implementation of a Bayesian joint model by means of the JMbayes package in R. Other models such as competing risks joint models and some specialised software functions are introduced in Section 7 and further described in Section S4. In Section 8, we conclude with a discussion of our findings.

\section{Modelling Framework}

Joint models are useful within a few different scenarios. The main interest can be in the longitudinal process with an event causing informative dropout. Interest could be equally on a longitudinal and a survival process that are associated. A third common scenario occurs when a time-to-event process is modelled with a longitudinal covariate measured intermittently and with error. Such data require joint modelling to fully capture the association and reduce bias.

Consider $i=1, \ldots, N$ subjects with repeated longitudinal measurements and a terminal event measured. The repeated measurements and event time are assumed to be associated. Each subject $i$ has $n_{i}$ measurements taken at different times with the intervals between measurements possibly differing. The total number of measurements is $n=\sum_{i=1}^{N} n_{i}$.

\subsection{Longitudinal Submodel}

Let $Y_{i}(t)$ denote the longitudinal process for subject $i$ at time $t$. We assume there is a true underlying process, or trajectory, $m_{i}(t)$ from which $Y_{i}(t)$ is measured with error $e_{i}(t)$, $Y_{i}(t)=m_{i}(t)+e_{i}(t)$. In practice, we do not observe the longitudinal process at all times. Instead for subject $i$, we observe $Y_{i}(t)$ at $n_{i}$ times $\left(t_{i 1}, \ldots, t_{i n_{i}}\right)$. Let $Y_{i}=\left(Y_{i 1}, \ldots, Y_{i n_{i}}\right)=$ $\left(Y_{i}\left(t_{i 1}\right), \ldots, Y_{i}\left(t_{i n_{i}}\right)\right)$ be the vector of observations. The trajectory $m_{i}(t)$ is modelled with fixed (possibly time dependent) covariates, $X_{1 i j}$ and parameter coefficients $\beta$ and random effects $b_{i}$ with possibly time-dependent covariates $Z_{i j}$. The longitudinal submodel is shown in (1).

$$
\begin{aligned}
Y_{i j} & =m_{i}\left(t_{i j}\right)+e_{i}\left(t_{i j}\right)=X_{1 i j} \beta+Z_{i j} b_{i}+e_{i j}, \\
\text { with } e_{i} & =\left(e_{i 1}, e_{i 2}, \ldots, e_{i n_{i}}\right) \sim N\left(0, \sigma^{2} I_{n_{i} \times n_{i}}\right), \\
b_{i} & \sim N\left(0, \Sigma_{b}\right), i=1, \ldots, N, j=1, \ldots, n_{i}
\end{aligned}
$$

where $\sigma^{2}$ and $\Sigma_{b}$ are the dispersion parameters for the error and the random effects, respectively.

\subsection{Time-to-Event Submodel}

Let $T_{i}^{*}$ denote the event time for subject $i, C_{i}$ the censoring time, $T_{i}=\min \left\{T_{i}^{*}, C_{i}\right\}$ the observed time and $\delta_{i}=1\left(T_{i}^{*} \leq C_{i}\right)$ the event indicator. A common time-to-event submodel is a relative risk model of the form (2).

$$
\lambda_{i}\left(t \mid M_{i}(t)\right)=\lambda_{0}(t) \exp \left\{X_{2 i} \gamma+\alpha m_{i}(t)\right\}
$$


where $M_{i}(t)$ is the history of the longitudinal process up to $t, M_{i}(t)=\left\{m_{i}(s), 0 \leq s<t\right\}$. The matrix of fixed covariates, $X_{2 i}$, can include the same variables as $X_{i 1}$ in the longitudinal submodel but they do not necessarily overlap. The trajectory $m_{i}(t)$ from the longitudinal model is included to link the two processes and $\alpha$ measures the strength of this association. While proportional hazards models are common, accelerated failure time and other survival models have been implemented (Mccrink et al., 2013; Tseng et al., 2005; Rizopoulos, 2012, p. 137).

The model form in (2) will be referred to as the current value form since the association is through the current value of the longitudinal trajectory, $m_{i}(t)$, on the right-hand side of the equation. This form is most often used when the survival time is of interest and the longitudinal process is thought of as a time-dependent covariate measured with error. In $(2), \lambda_{i}(t)$ is the event hazard and $\lambda_{0}(t)$ is the baseline hazard. In ordinary Cox, regression $\lambda_{0}(t)$ is left unspecified, avoiding restrictions that come from specifying a parametric form for the baseline hazard (Yuen \& Mackinnon, 2016). However if an unspecified hazard is applied in a joint model, it has been shown that standard errors of the parameter estimates can be underestimated (Hseih et al., 2006). This can be remedied by estimating the standard errors with an additional method such as bootstrapping (Rizopoulos, 2010; Yuen \& Mackinnon, 2016), profile likelihood (Murphy \& van der Vaart, 2000) or numerically differentiating the EM operator (Xu et al., 2014). To avoid this issue, parametric but flexible functions are often used for $\lambda_{0}(t)$, such as piecewise constant or spline models (Rizopoulos, 2012, p. 53). These flexible functions can sufficiently approximate the baseline hazard and has been noted as the preferred choice for $\lambda_{0}(t)$ by some (Rizopoulos, 2010; Yuen \& Mackinnon, 2016; Rizopoulos, 2012, p. 53).

Alternatively, the submodels can be linked through a shared parameters model. This is often used when the longitudinal process is the main interest with informative censoring or when the focus is on both processes equally. Assuming a longitudinal submodel as in (1), a survival submodel as shown in (3) is common

$$
\lambda_{i}(t)=\lambda_{0}(t) \exp \left\{X_{i 2} \gamma+\alpha Z_{2 i}(t) b_{i}\right\}
$$

where $b_{i}$ is the same vector of random effects as in (1) and $\alpha$ again measures the association. A typical example is a random coefficients model where $Z_{2 i}(t) b_{i}=b_{0 i}+b_{1 i} t$ with $b_{0 i}$ and $b_{1 i}$ correlated, often multivariate normal (Wulfsohn \& Tsiatis, 1997). The association can be generalised so that $\alpha Z_{2 i}(t) b_{i}=\alpha_{0} b_{0 i}+\alpha_{1} b_{1 i} t$ and $\alpha_{0}$ need not equal $\alpha_{1}$ (Mccrink et al., 2013).

\subsection{Estimation}

It is commonly assumed that conditionally given the random effects, the longitudinal and time-to-event outcomes are independent and so are the longitudinal measurements taken on a single subject. Denoting the set of all parameters by $\theta=(\beta, \gamma, \alpha)$, the log-likelihood contribution from subject $i$ is

$$
l_{i}(\theta)=\log \int\left(\prod_{j=1}^{n_{i}} f\left(y_{i j} \mid b_{i} ; \theta\right)\right) \lambda_{i}\left(T_{i} \mid b_{i} ; \theta\right)^{\delta_{i}} S\left(T_{i} \mid b_{i} ; \theta\right) f\left(b_{i} ; \theta\right) d b_{i},
$$

where $f(\cdot)$ denotes the density function and $S(\cdot)$ denotes the survival function. There is generally no closed form solution to the likelihood equations. Numerical integration and optimisation techniques, such as an Expectation-Maximization (EM) algorithm treating the random effects as missing data, are used in practice. Due to high-dimensional integration and potential correlation induced by the random effects, the process of convergence can be slow. Other methods include Newton-type or hybrid-EM and quasi-Newton algorithms (Rizopoulos, 2012, p.64; Henderson et al., 2000; Hseih et al., 2006; Tsiatis \& Davidian, 2004; Wulfsohn \& Tsiatis, 1997; Yu et al., 2004). 


\section{An Application}

We explore the available software platforms through analysis of a dataset of 176 adrenal cancer patients at the University of Michigan Comprehensive Cancer Center diagnosed between 1983 and 2011. Multiple measurements of adrenal tumour characteristics were collected from periodic computed tomography scans. Time until death or last follow-up was also collected. We selected a single scan measurement as the longitudinal response variable. The longitudinal outcome chosen was psoas density in Hounsfield units measured using the density of pixels in the scan (Holcombe et al., 2016). A previous study with similar data describes how psoas muscle density and size can be measures of patient frailty, and scan measurements can be associated with survival (Miller et al., 2012). Baseline covariates used were age, cancer stage and tumour grade. For simplicity, we performed a complete case analysis. There were 160 patients with psoas density measurements and all baseline covariates. There are between 1 and 45 scans for each patient with a mean of 5.5 and median of three scans. In this group, 100 patients died. Time until death or censoring fell between 0.1 and 17.9 years with a median of 2.4 years. Table 1 summarises the relevant variables. Joint models are fit to the data using three major softwares, namely, R, SAS and Stata. Both the current value (2) and the shared parameter (3) forms are implemented.

First, a current-value joint model was fit to this data using JM in R, SAS and Stata. The longitudinal submodel included a random intercept and slope in each software.

$$
\begin{aligned}
\text { PsoasDensity }_{i j}= & m_{i}\left(t_{i j}\right)+e_{i j}=\beta_{0}+\beta_{1} t_{i j}+\beta_{2} \text { Age }_{i}+\beta_{3} \text { Stage }_{i}+\beta_{4} \text { TumourGradeHigh }_{i} \\
& +\beta_{5} \text { TumourGradeUnknown }_{i}+b_{0 i}+b_{1 i} t_{i j}+e_{i j} .
\end{aligned}
$$

Survival submodels with flexible baseline hazards were chosen since the true model is unknown. Stata were unable to fit the data with a flexible baseline hazard so a specific parametric (Weibull) hazard was used. Specifically, the softwares fit survival submodels with the form

$$
\begin{aligned}
\lambda_{i}(t)= & \lambda_{0}(t) \exp \left(\gamma_{1} \text { Age }_{i}+\gamma_{2} \text { Stage }_{i}+\gamma_{3} \text { TumourGradeHigh }_{i}\right. \\
& \left.+\gamma_{4} \text { TumourGradeUnknown }_{i}+\alpha m_{i}(t)\right),
\end{aligned}
$$

with baseline hazards

R JM PWC: $\lambda_{0}(t) \sim$ Piecewise constant function

SAS \%JM PWC: $\lambda_{0}(t) \sim$ Piecewise constant function

Stata stjm Weib: $\lambda_{0}(t) \sim$ Weibull.

Table 1. Description of adrenal cancer data.

\begin{tabular}{lccc}
\hline Variable & Mean or count & SD or \% \\
\hline Psoas density & & 54.4 & 8.5 \\
Scan time (years) & & 3.1 & 3.2 \\
Age & & 46.1 & 13.6 \\
Stage & 1 or 2 & 71 & $44.4 \%$ \\
& 3 or 4 & 89 & $55.6 \%$ \\
Tumour grade & Low & 79 & $49.4 \%$ \\
& High & 58 & $36.3 \%$ \\
& Unknown & 23 & $14.4 \%$ \\
\hline
\end{tabular}


For SAS and R JM, the program defaults were used to create the flexible baseline hazards. Both procedures by default use six equally spaced internal knots to partition the observed event times. For the SAS model, the random effects are assumed uncorrelated because we found fitting issues with an unstructured matrix (Garcia-Hernandez \& Rizopoulos, 2015).

A two-stage model was fit for comparison.

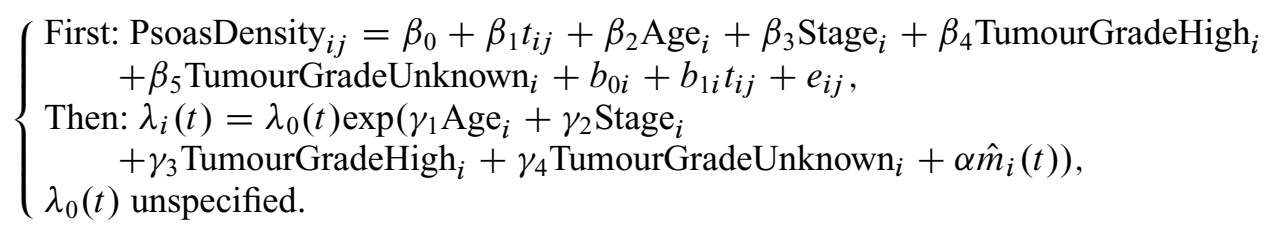

Bootstrapping was used to estimate the coefficients and bias-corrected bootstrapped confidence intervals (Efron \& Tibshirani, 1993), as shown in Table 2.

We also fit a shared parameter model as in (3) with joineR, SAS and Stata. The longitudinal submodel included a random intercept in joineR and Stata, while the default in SAS included a random slope. The model fit is

$$
\begin{gathered}
\text { PsoasDensity }_{i j}=\beta_{0}+\beta_{1} t_{i j}+\beta_{2} \text { Age }_{i}+\beta_{3} \text { Stage }_{i}+\beta_{4} \text { TumourGradeHigh }_{i} \\
+\beta_{5} \text { TumourGradeUnknown }_{i}+b_{i}+e_{i j} \\
\lambda_{i}(t)=\lambda_{0}(t) \exp \left(\gamma_{1} \text { Age }_{i}+\gamma_{2} \text { Stage }_{i}+\gamma_{3} \text { TumourGradeHigh }_{i}\right. \\
\left.+\gamma_{4} \text { TumourGradeUnknown }_{i}+\alpha b_{i}\right),
\end{gathered}
$$

with baseline hazards

R joineR: $\lambda_{0}(t)$ Unspecified

SAS \%JM PWC: $\lambda_{0}(t) \sim$ Piecewise constant function

Stata stjm Weib: $\lambda_{0}(t) \sim$ Weibull.

The findings are contrasted with a shared parameter two-stage model similar to that in (6) but with $\alpha \hat{m}_{i}(t)$ replaced with $\alpha b_{i}$.

Table 2. Current-value joint model parameter estimates and bias-corrected bootstrapped confidence intervals for the adrenal data.

\begin{tabular}{lccll}
\hline Variable & \multicolumn{1}{c}{ Two-stage $^{*}$} & JM PWC & SAS \%JM PWC & Stata stjm Weib \\
\hline$N$ & 89 & 87 & 100 & 100 \\
Intercept & $64.4(59.5,68.2)$ & $64.6(599.2,68.3)$ & $65.4(59.8,69.7)$ & $64.6(60.6,68.6)$ \\
Time & $-0.5(-1.4,-0.1)$ & $-0.6(-1.7,-0.2)$ & $-0.9(-2.7,-0.4)$ & $-0.6(-1.9,-0.3)$ \\
Age & $-0.2(-0.3,-0.1)$ & $-0.2(-0.3,-0.1)$ & $-0.2(-0.3,-0.1)$ & $-0.2(-0.4,-0.2)$ \\
Stages 3 or 4 & $-0.6(-1.7,0.2)$ & $-0.6(-1.7,0.3)$ & $-0.6(-1.8,0.05)$ & $-0.6(-2.2,-0.1)$ \\
Tumour grade: High & $-0.6(-3.8,2.2)$ & $-0.7(-4.1,2.1)$ & $-0.7(-4.1,2.1)$ & $-0.7(-3.6,2.1)$ \\
Tumour grade: unknown & $-2.9(-7.5,0.3)$ & $-3.2(-8.4,-0.6)$ & $-3.1(-8.0,0.2)$ & $-3.1(-8.5,-0.4)$ \\
Surv-age & $0.1(-0.009,0.6)$ & $0.002(-0.01,0.06)$ & $-0.009(-0.03,0.008)$ & $-0.01(-0.03,0.001)$ \\
Surv-stages 3 or 4 & $0.7(-0.06,3.4)$ & $0.2(0.02,0.3)$ & $0.2(-0.01,0.3)$ & $0.2(-0.06,0.4)$ \\
Surv-tumour grade: high & $1.3(-3.6,4.2)$ & $0.6(0.004,1.0)$ & $0.5(0.06,1.1)$ & $0.4(-0.04,1.1)$ \\
Surv-tumour grade: unknown & $3.4(0.4,12.0)$ & $1.0(0.3,1.5)$ & $0.9(0.2,1.7)$ & $1.0(0.2,1.9)$ \\
Association & $0.8(0.1,3.0)$ & $-0.07(-0.1,-0.04)$ & $-0.1(-0.2,-0.06)$ & $-0.09(-0.1,-0.04)$ \\
\hline
\end{tabular}

$N$ is the number of models in 100 bootstraps that successfully converged.

${ }^{*}$ Two-stage intervals are the 2.5 and 97.5 quantiles of the bootstrapped values, not bias-corrected. 
Comparing Tables 2 and 3, the results are similar for the current-value and shared parameters models. Nearly all joint models estimate the coefficients of the longitudinal submodel very similarly. Time has a significant negative value showing that psoas density tends to decrease over time, which fits with previous knowledge (Miller et al., 2012). Older patients tend to have less psoas density. Stages 3 or 4 and high tumour grade tend to decrease the average psoas density compared to lower stages and grade, but these are not significant in any software or models. There are more differences in the results of the survival submodel since each software fits the survival model differently. The effects are estimated to be larger in the two-stage currentvalue models than in the true joint models. Age does not have much of an effect on death hazard. Most software and model forms find higher stage to slightly increase the death hazard, but significance depends on the software. There is a small and significant positive coefficient for high tumour stage. Finally, each software except the current-value two-stage model found a small but significant negative association. Log-likelihoods are shown in Table 4 and are similar for all software and both model forms.

\section{Assessment of Implementation Platform}

Fitting a joint model can be computationally intensive. As indicated in the data analysis section, there are a few software packages that are designed to fit such models in an efficient manner. However, the class of such models is quite large, and the softwares use different iterative algorithms for estimation and integration. It is thus important to compare and contrast the implementation platforms to assess and appraise the performance of the competing tools. Such assessment is difficult to make on the basis of fits to few specific datasets. We undertake this

Table 3. Shared parameter joint model parameter estimates and bootstrapped confidence intervals for the adrenal data.

\begin{tabular}{lllll}
\hline Variable & \multicolumn{1}{c}{ Two-stage $^{*}$} & \multicolumn{1}{c}{ joineR } & SAS \%JM PWC & Stata stjm Weib \\
\hline$N$ & 100 & 100 & 100 & 100 \\
Intercept & $63.7(59.5,67.7)$ & $63.1(58.3,67.1)$ & $64.5(60.0,68.5)$ & $64.4(59.5,68.1)$ \\
Time & $-0.3(-0.6,-0.08)$ & $-0.4(-0.7,-0.2)$ & $-0.6(-1.4,-0.3)$ & $-0.6(-1.2,-0.3)$ \\
Age & $-0.2(-0.3,-0.1)$ & $-0.2(-0.3,-0.1)$ & $-0.2(-0.3,-0.1)$ & $-0.2(-0.3,-0.09)$ \\
Stage 3 or 4 & $-0.6(-1.7,0.1)$ & $-0.7(-1.6,0.1)$ & $-0.6(-1.7,0.09)$ & $-0.6(-2.3,0.3)$ \\
Tumour grade: high & $-0.6(-3.6,2.0)$ & $0.6(-2.0,3.7)$ & $-0.7(-3.7,2.1)$ & $-0.6(-4.0,2.2)$ \\
Tumour grade: unknown & $-3.2(-9.1,1.6)$ & $-2.9(-7.7,3.3)$ & $-3.1(-8.1,-0.09)$ & $-3.2(-7.5,-0.3)$ \\
Surv-age & $0.01(-0.003,0.03)$ & $0.01(-0.004,0.02)$ & $0.01(-0.003,0.03)$ & $0.006(-0.02,0.02)$ \\
Surv-stages 3 or 4 & $0.2(0.06,0.4)$ & $0.2(0.01,0.4)$ & $0.2(0.04,0.4)$ & $0.2(-0.02,0.5)$ \\
Surv-tumour grade: high & $0.5(-0.01,1.0)$ & $-0.5(-1.0,0.02)$ & $0.6(0.04,1.2)$ & $0.4(-0.04,1.1)$ \\
Surv-tumour grade: unknown & $1.1(0.6,1.8)$ & $0.7(-0.2,1.3)$ & $1.2(0.4,1.8)$ & $1.3(0.4,2.1)$ \\
Association & $-0.09(-0.1,-0.06)$ & $-0.09(-0.1,-0.05)$ & $-0.1(-0.1,-0.06)$ & $-0.09(-0.1,-0.05)$ \\
\hline
\end{tabular}

$N$ is the number of models in 100 bootstraps that successfully converged.

${ }^{*}$ Intervals are the 2.5 and 97.5 quantiles of the bootstrapped values, not bias-corrected.

Table 4. Log-likelihood values for the adrenal data models.

\begin{tabular}{lcc}
\hline & Current-value & Shared parameter \\
\hline JM PWC & -3130.6 & NA \\
joineR & NA & -3392.1 \\
SAS \%JM PWC & -3147.5 & -3147.0 \\
Stata stjm Weib & -3100.9 & -3083.3 \\
\hline
\end{tabular}


evaluation with simulations using various statistical software modules available for fitting joint models. In order to lay the groundwork for the simulation, we first need to briefly describe the available software packages. All packages described in this section implement (1) and either (2) or (3) under a frequentist framework.

\subsection{JM Package in $R$}

The JM package in the $\mathrm{R}$ language was designed for fitting joint models with the jointModel() function. A full description of this package and the jointmodel() function are available in the Comprehensive R Archive Network (CRAN) documentation (Rizopoulos, 2016a).

The main arguments for the jointModel () function include the output from a linear mixed model (from the R function lme () ) (Pinheiro et al., 2016) and the output from a Cox proportional hazard model (usually from the R function coxph ( )) (Therneau \& Lumley, 2016). Using these separate model fits, jointModel () fits a corresponding current-value joint model with submodels having the same covariates and forms as in the separate models and with the additional association added to the survival submodel.

The jointmodel() function fits three different model forms using the parameterisation argument. The default is parameterisation = "value" and fits the current value model in (2). For parameterisation = "slope", the survival submodel is linked to the longitudinal process through the slope of the trajectory as in (7).

$$
\lambda_{i}\left(t \mid M_{i}(t)\right)=\lambda_{0}(t) \exp \left\{X_{2 i} \gamma+\alpha m_{i}^{\prime}(t)\right\} .
$$

The final option is parameterisation = "both" which fits a model with both the current value and the slope of the trajectory in the survival submodel, namely, $\lambda_{i}\left(t \mid M_{i}(t)\right)=$ $\lambda_{0}(t) \exp \left\{X_{2 i} \gamma+\alpha_{1} m_{i}(t)+\alpha_{2} m_{i}^{\prime}(t)\right\}$.

The method argument specifies the form of the baseline hazard, the form of the model and the method of numerical integration. The available options are weibull-PH-aGH, piecewise-PH-aGH, spline-PH-aGH, weibull-AFT-aGH, Cox-PH-aGH and ch-Laplace. The method ch-Laplace uses the fully exponential Laplace approximation described by Rizopoulos et al. (2009). All other options follow a similar format. The first word describes the baseline hazard: weibull uses a Weibull baseline hazard, piecewise a piece-wise constant baseline hazard, spline a B-spline approximation and Cox an unspecified baseline risk. Options with PH fit a proportional hazards survival submodel. There is one option for an accelerated failure time model using a Weibull baseline (weibull-AFT-GH). Methods ending in aGH use pseudo-adaptive Gauss-Hermite quadrature for integral approximation, where the quadrature knots are reassigned once after the first iteration (Rizopoulos, 2010). Each method can instead end in GH which uses standard Gauss-Hermite quadrature. Adaptive quadrature is generally preferred due to a reduced computational load using fewer quadrature points while still achieving error on the same order of magnitude as with the standard quadrature (Yuen \& Mackinnon, 2016). Quasi-Newton techniques are used if EM iterations do not achieve convergence quickly. Only EM is used with the unspecified baseline hazard. Stratification is allowed only with method= "spline-PH-aGH" or method= "spline-PH-GH".

The jointModel ( ) function allows for some extensions such as a competing risks model with the CompRisk argument. Other extensions can be formulated with the interFact and derivform options as described in Rizopoulos (2010) and Rizopoulos (2016a). 


\section{2 joineR Package in $R$}

The joineR package was created to analyse longitudinal studies, possibly with an event time causing informative censoring. Full description of this package is in the CRAN documentation (Philipson et al., 2012). The joint ( ) function fits a joint model and requires data in a specific format that is the output from the function jointdata () in the same package. The user supplies the joint () function with the data and a formula object specifying the form for each of the longitudinal and survival submodels. This function fits a shared parameter joint model as in (1) and (3) with an unspecified baseline hazard. This function is not capable of specifying a parametric form for the baseline hazard. The model argument determines the shared random effects. A random slope and intercept model is the default, whereas model= "int" specifies a random intercept only, and model= "quad" adds a quadratic time effect to the intercept and slope. The default settings fit a common association when there is more than one random effect terms $\left(\alpha Z_{2 i}(t) b_{i}=\alpha b_{0 i}+\alpha b_{1 i} t\right)$. A separate association, as discussed in Section 2.2, can be implemented using the sepassoc=TRUE option $\left(\alpha Z_{2 i}(t) b_{i}=\alpha_{0} b_{0 i}+\alpha_{1} b_{1 i} t, \alpha_{0} \neq \alpha_{1}\right)$. This function also uses an EM algorithm for estimation with some options available for control of this approximation. Since not specifying a baseline hazard may lead to underestimated standard errors, the joineR package includes a separate function to calculate bootstrap standard errors for the joint model (jointSE ( )).

\section{$4.3 \%$ JM Macro in SAS}

The \%JM macro in the SAS language was written to fit several joint models in several possible forms. A full description of the macro was written by Garcia-Hernandez and Rizopoulos (2015). The \%JM macro allows the longitudinal data to be fit to varying outcome types. The longitudinal data can conform to a normal, binomial or Poisson distribution, corresponding to continuous, categorical or count outcomes, respectively. These can be specified with the LongiType option. The longitudinal model can also fit different random effects with the Longi TimeModel option, including a linear random intercept and slope model and multiple random splines. There are many options for the baseline hazard of the survival submodel using the EventModel option, such as exponential, Weibull, piecewise and several spline options. The survival submodel can include stratification factors using the Eventstrata argument. The association can be set with the SharedParam argument with the options including current value as in (2), slope (equivalent to our model (3) with $Z_{2 i}(t) b_{i}=b_{0 i}+b_{1 i} t$ ), cumulative $\left(\lambda_{i}\left(t \mid M_{i}(t)\right)=\lambda_{0}(t) \exp \left\{X_{2 i} \gamma+\alpha \int_{0}^{t} m_{i}(s) d s\right\}\right)$ and 'coefficients' in which the user can specify which random effects from the longitudinal submodel should be included in the survival submodel. Multiple associations can be used at the same time. The SharedCoefficients and SharedLongitTerm arguments can be used to create even more joint model parameterisations. Estimation is carried out by the PROC NLMIXED procedure which is described in Section S4.1.

\section{4 stjm Command in Stata}

In Stata, joint models can be fit with the stjm command. A detailed description is given by Crowther et al. (2013). A linear mixed model and a proportional hazards model can be fit as the submodels with several association structures available. The association between submodels can be of the current value or the current slope form, similar to the JM package in R. The association can also be through shared parameters with or without covariates. Estimation is carried out by Newton-Ralphson method, and numerical integration is implemented with standard or 
adaptive Gauss-Hermite quadrature. We found Stata to have more difficulties in fitting models with various parameter values in our simulations.

\subsection{Comparison of Software Functionality}

In summary, all three major statistical softwares R, SAS and Stata fit joint models with comparable functionality. A detailed list of available options is provided in Table 5. All software will

Table 5. Overview of available functionalities for joint modelling in each software; ' $x$ ' means the software in that column has the feature in the corresponding row.

\begin{tabular}{|c|c|c|c|c|c|c|}
\hline Functionality & $\begin{array}{c}\text { JM R } \\
\text { package }\end{array}$ & $\begin{array}{l}\text { joineR R } \\
\text { package }\end{array}$ & $\begin{array}{c}\% \mathrm{JM} \\
\text { SAS macro }\end{array}$ & $\begin{array}{c}\% \text { JMfit } \\
\text { SAS macro }\end{array}$ & $\begin{array}{l}\text { stjm Stata } \\
\text { Command }\end{array}$ & $\begin{array}{c}\text { JMbayes R } \\
\text { package }\end{array}$ \\
\hline \multicolumn{7}{|l|}{ Longitudinal submodel } \\
\hline Gaussian linear model & $\mathrm{x}$ & $\mathrm{x}$ & $\mathrm{x}$ & $\mathrm{x}$ & $\mathrm{x}$ & $\mathrm{x}$ \\
\hline Generalised linear models & $\mathrm{x}$ & & $\mathrm{x}$ & & & $\mathrm{x}$ \\
\hline Covariance matrix options & & & $\mathrm{x}$ & & $\mathrm{x}$ & \\
\hline \multicolumn{7}{|l|}{ Survival submodel } \\
\hline Relative risk model & $\mathrm{x}$ & $\mathrm{x}$ & $\mathrm{x}$ & $\mathrm{x}$ & $\mathrm{x}$ & $\mathrm{x}$ \\
\hline AFT Weibull model & $\mathrm{x}$ & & & & & \\
\hline Unspecified baseline hazard & $\mathrm{x}$ & $\mathrm{x}$ & & & & \\
\hline Piecewise baseline hazard & $\mathrm{x}$ & & $\mathrm{x}$ & $\mathrm{x}$ & & \\
\hline Spline baseline hazard & $\mathrm{x}$ & & $\mathrm{x}$ & $\mathrm{x}$ & $\mathrm{x}$ & $\mathrm{x}$ \\
\hline \multicolumn{7}{|l|}{ Association } \\
\hline Current value assoc. & $\mathrm{x}$ & & $\mathrm{x}$ & & $\mathrm{x}$ & $\mathrm{x}$ \\
\hline Current slope assoc. & $\mathrm{x}$ & & $\mathrm{x}$ & & $\mathrm{x}$ & $\mathrm{x}$ \\
\hline Random intercept assoc. & & $\mathrm{x}$ & $\mathrm{x}$ & $\mathrm{x}$ & $\mathrm{x}$ & $\mathrm{x}$ \\
\hline Random slope association & & $\mathrm{x}$ & $\mathrm{x}$ & $\mathrm{x}$ & $\mathrm{x}$ & $\mathrm{x}$ \\
\hline Separate associations & & $\mathrm{x}$ & & $\mathrm{x}$ & & $\mathrm{x}$ \\
\hline Interactions in associations & $\mathrm{x}$ & & $\mathrm{x}$ & $\mathrm{x}$ & $\mathrm{x}$ & $\mathrm{x}$ \\
\hline \multicolumn{7}{|l|}{ Model fit options } \\
\hline Lagged effects & $\mathrm{x}$ & & $\mathrm{x}$ & & & $\mathrm{x}$ \\
\hline Competing risks models & $\mathrm{x}$ & & $\mathrm{x}$ & & & \\
\hline Initial value options & $\mathrm{x}$ & & $\mathrm{x}$ & $\mathrm{x}$ & & $\mathrm{x}$ \\
\hline Stratification in survival submodel & $\mathrm{x}$ & & $\mathrm{x}$ & & & \\
\hline Piecewise/spline hazard customisation & $\mathrm{x}$ & & $\mathrm{x}$ & $\mathrm{x}$ & & $\mathrm{x}$ \\
\hline \multicolumn{7}{|l|}{ Estimation options } \\
\hline EM only & $\mathrm{x}$ & $\mathrm{x}$ & & & & \\
\hline Quasi-Newton & $\mathrm{x}$ & & $\mathrm{x}$ & $\mathrm{x}$ & & \\
\hline Bayesian MCMC & & & & & & $\mathrm{x}$ \\
\hline Number of iteration control & $\mathrm{x}$ & $\mathrm{x}$ & $\mathrm{x}$ & $\mathrm{x}$ & $\mathrm{x}$ & $\mathrm{x}$ \\
\hline Convergence tolerance control & $\mathrm{x}$ & $\mathrm{x}$ & $\mathrm{x}$ & $\mathrm{x}$ & $\mathrm{x}$ & \\
\hline Piecewise/spline knots control & $\mathrm{x}$ & & $\mathrm{x}$ & $\mathrm{x}$ & & $\mathrm{x}$ \\
\hline Quadrature points control & $\mathrm{x}$ & $\mathrm{x}$ & $\mathrm{x}$ & $\mathrm{x}$ & $\mathrm{x}$ & \\
\hline Adaptive Gauss-Hermite quadrature & & & $\mathrm{x}$ & $\mathrm{x}$ & $\mathrm{x}$ & \\
\hline Pseudo-adaptive Gauss-Hermite quadrature & $\mathrm{x}$ & & & & & \\
\hline Laplace approximation & $\mathrm{x}$ & & $\mathrm{x}$ & & & \\
\hline Gauss-Kronrod rule & $\mathrm{x}$ & & $\mathrm{x}$ & & $\mathrm{x}$ & \\
\hline \multicolumn{7}{|l|}{ Other options } \\
\hline $\mathrm{AIC}$ or $\mathrm{BIC}$ & & & $\mathrm{x}$ & $\mathrm{x}$ & & $\mathrm{x}$ \\
\hline Plotting & $\mathrm{x}$ & $\mathrm{x}$ & & & $\mathrm{x}$ & $\mathrm{x}$ \\
\hline Predictions & $\mathrm{x}$ & & & & & $\mathrm{x}$ \\
\hline Approx SE default & $\mathrm{x}$ & & $\mathrm{x}$ & $\mathrm{x}$ & $\mathrm{x}$ & \\
\hline Bootstrap SE option & & $\mathrm{x}$ & $\mathrm{x}$ & & $\mathrm{x}$ & \\
\hline
\end{tabular}


fit a Gaussian longitudinal submodel as in (1). Some software will fit a generalised linear model where the longitudinal outcome $Y$ has a non-Gaussian distribution such as binomial or Poisson. Each software will also fit a standard proportional hazards survival submodel modelling the hazard function as in (2) or (3). Only JM in R will fit a survival model in an accelerated failure time (AFT) framework. Available association options are listed including current value (2), random intercept or slope (3) and separate associations like we saw in Section 4.2. Model fit options describe different forms of the survival submodels that may be available such as a competing risks model, lagged effects or stratification. Estimation options include choices for the model fitting algorithm and numerical integration. Finally, some other possibly useful options in the software are listed including built-in AIC or BIC calculations, plotting, predictions and standard error calculations.

\section{Simulations}

The joint modelling software were compared through simulation. Data were generated under three scenarios, namely, a random intercept only and a random intercept and slope model in the current-value form and a random intercept shared-parameters model. Each scenario includes $N=500$ subjects and 100 simulated datasets. Data were generated in $\mathrm{R}$ version 3.2.2 and simulations were run in Windows 7 on a $3.2 \mathrm{GHz}$ Intel Core i5 processor.

\subsection{Scenario 1}

Data for the current-value association random intercept only joint model, which we will call Scenario 1, were generated as follows. The longitudinal data were generated from the model

$$
Y_{i j}=m_{i}\left(t_{i j}\right)+e_{i j}=\beta_{0}+\beta_{1} t_{i j}+\beta_{2} X_{1 i}+\beta_{3} X_{2 i}+b_{i}+e_{i j} .
$$

We have two covariates in our joint model, a binary group indicator $X_{1}$ and a continuous $X_{2}$. Measurement times are between 0 and 3 years. The random effect and measurement error are normally distributed and independent. Survival times $T_{i}^{*}$ were generated from a relative risk model.

$$
\lambda_{i}(t)=\exp \left(\gamma_{0}+\gamma_{1} X_{1 i}+\gamma_{2} X_{2 i}+\alpha m_{i}(t)\right) .
$$

(See the Section S1 for details on generating the survival times). The parameter values used are shown thereafter.

$$
\begin{aligned}
& X_{1 i} \sim \text { Bernoulli(0.5), } \\
& X_{2 i} \sim N(0,1), \\
& \left(t_{i 1}, t_{i 2}, \ldots, t_{i 7}\right)=(0,0.5,1,1.5,2,2.5,3) \text {, } \\
& e_{i j} \sim N(0,4) \text {, } \\
& b_{i} \sim N(0,2.25) \\
& \begin{array}{ll}
\beta_{0}=0.6 & \gamma_{0}=-4.4 \\
\beta_{1}=0.2 & \gamma_{1}=0.07 \\
\beta_{2}=-0.1 & \gamma_{2}=0.2 \\
\beta_{3}=0.4 & \alpha=0.5
\end{array} .
\end{aligned}
$$

Independent censoring times $C_{i}$ were drawn from a uniform distribution, $C_{i} \sim \operatorname{Unif}(0,3.25)$, and the observed time $T_{i}$ is the minimum, $T_{i}=\min \left(T_{i}^{*}, C_{i}\right)$. Any longitudinal measurements $Y_{i j}$ at times after the observed time $T_{i}$ (where $t_{i j}>T_{i}$ for subject $i$ ) were dropped.

The R JM, joineR, Stata stjm and two-stage software fit (8) to the longitudinal part. The SAS macro by default fits a model with a random intercept and slope. The \% JM macro has no built-in option for an intercept only association. Therefore, we fit a misspecified longitudinal model in SAS, replacing $b_{i}$ with $b_{0 i}+b_{1 i} t_{i j}$ in (8). An unstructured covariance matrix was used 
for the SAS PWC model. For the SAS Weibull model, the default diagonal covariance matrix was used to achieve convergence.

For the survival part, a current-value association was used if available. The R JM package, Stata command and the \% JM macro in SAS fit a current-value survival submodel as specified in (9). The joineR package only fits joint models with the shared parameters association conforming to the structure in (10).

$$
\lambda_{i}(t)=\lambda_{0}(t) \exp \left(X_{1 i} \gamma_{1}^{*}+X_{2 i} \gamma_{2}^{*}+\alpha b_{i}\right) .
$$

This amounts to a re-parameterisation of the current-value model where the coefficients $\gamma_{1}^{*}$ and $\gamma_{2}^{*}$ in the joineR survival submodel converge to combinations of the true coefficients from both submodels, specifically $\gamma_{1}^{*}=\gamma_{1}+\alpha \beta_{2}$ and $\gamma_{2}^{*}=\gamma_{2}+\alpha \beta_{3}$. For all results, the joineR coefficients will be compared to these combinations. See Section S2 for more on this reparameterisation.

The baseline hazards used for these software are in (11). Pseudo-adaptive Gauss-Hermite (GH) quadrature is implemented in R JM Weib, PWC and Spl. For the model with an unspecified baseline hazard (R JM Unspec NA), we used the standard (nonadaptive) GH quadrature, since we found using the adaptive algorithm led to poor convergence. In order to investigate whether there is a difference in the nonadaptive (NA) versus the pseudo-adaptive GH quadrature, we also ran models with each of Weibull, piecewise constant and spline based baseline hazards, using standard GH quadrature, labelled R JM Weib NA, R JM PWC NA and R JM Spl NA.

R JM Weib: $\lambda_{0}(t) \sim$ Weibull

R JM PWC: $\lambda_{0}(t) \sim$ Piecewise constant function

R JM Spl: $\log \left(\lambda_{0}(t)\right) \sim$ B-spline approximation

R JM Unspec NA: $\lambda_{0}(t)$ unspecified

SAS \%JM Weib: $\lambda_{0}(t) \sim$ Weibull

SAS \%JM PWC: $\lambda_{0}(t) \sim$ Piecewise constant function

Stata stjm Weib: $\lambda_{0}(t) \sim$ Weibull

$\mathrm{R}$ joineR: $\lambda_{0}(t)$ unspecified

A two-stage model is also fit to compare this more simple technique to the true joint models. The two-stage model is fit as follows.

$$
\left\{\begin{array}{l}
\text { First: } Y_{i j}=\beta_{0}+\beta_{1} t_{i j}+\beta_{2} X_{1 i}+\beta_{3} X_{2 i}+b_{i}+e_{i j}, \\
\text { Then: } \lambda_{i}(t)=\lambda_{0}(t) \exp \left(\gamma_{1} X_{1 i}+\gamma_{2} X_{2 i}+\alpha \hat{m}_{i}(t)\right) .
\end{array}\right.
$$

We used the default options for numerical integration and for defining the flexible baseline hazard functions. Specifically, the R JM PWC and SAS \%JM PWC models both use a baseline hazard constructed from six equally spaced internal knots that partition the observed event times with the function being constant in between knots. For the R JM Spl model, the B-spline approximation is constructed with five internal knots.

Results are shown in Figure 1, plotting the bias of the estimated coefficients compared to the truth. Coverage probabilities are shown in Table 6. Tables with the bias and MSE for each parameter and the width of confidence intervals are in Supporting Information. Widths were calculated as the average of confidence interval lengths using a normal approximation, specifically the average of $2 * 1.96 *$ (standard error). Table 7 shows the run times for each software.

All software except Stata were able to fit models to a majority of the 100 datasets. Stata were only able to fit around one third of the datasets. Each software had relatively good coverage although Stata did not cover the intercept term well. The intervals around the association 
(a) Longitudinal Intercept

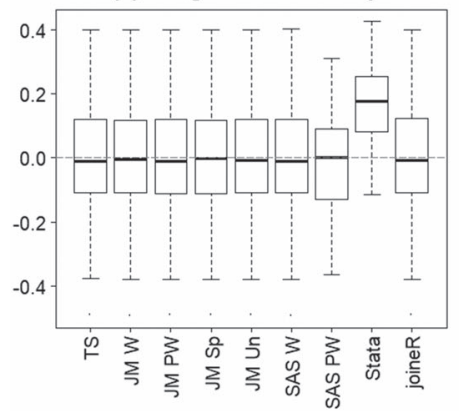

(d) Longitudinal X2

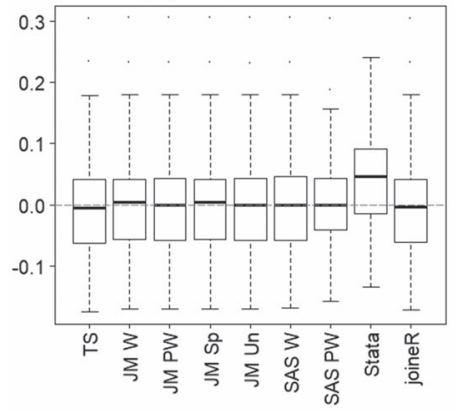

(g) Associations

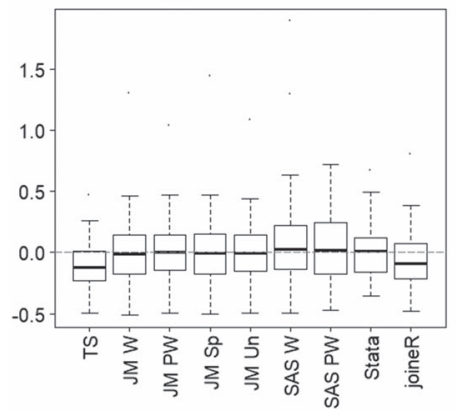

(b) Longitudinal Time

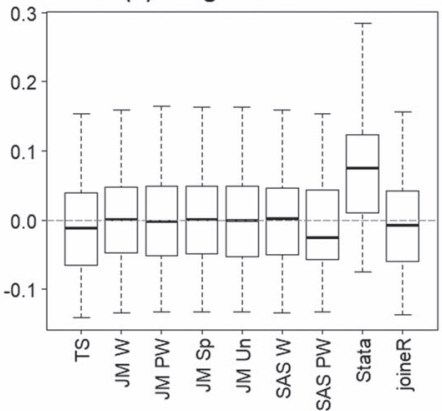

(e) Survival X1

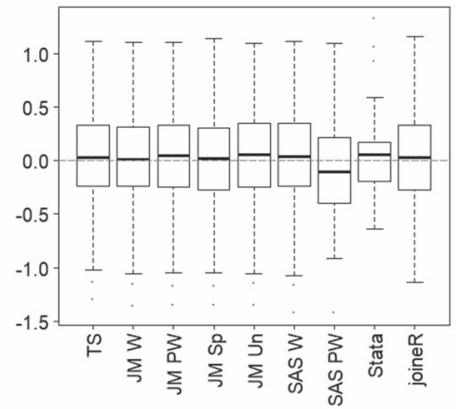

(h) Random Effects Variance

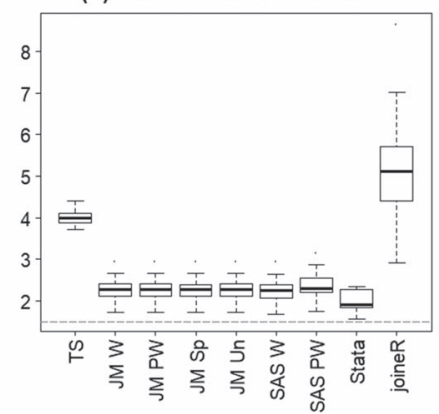

(c) Longitudinal $\mathbf{X} 1$

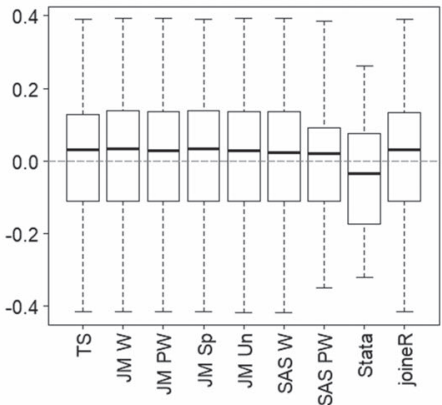

(f) Survival X2

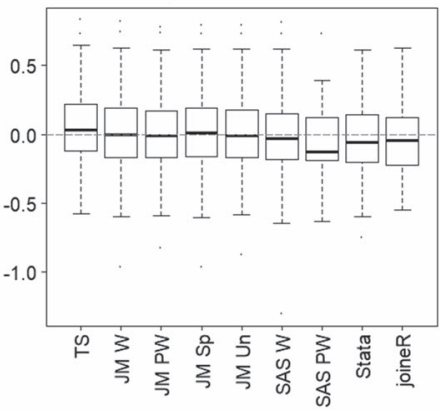

Figure 1. Boxplots showing the bias of the Scenario 1 estimates. Model names abbreviated as: TS $=$ TwoStage, JM W=R $J M$ Weibull, JM $P W=R$ JM Piece-Wise, JM Sp = R JM Spline, JM Un = R JM Unspecified NA, SAS W = SAS Weibull, SAS $P W=S A S$ Piece-Wise. Figure 1(h) plots the actual values estimated for the random effect variance with the dotted line at the true value.

parameter from R JM Unspec NA are narrower than the other R JM models. Stata tend to have a larger bias but smaller MSE than the other software leading to narrow confidence intervals. Every software overestimated the random effect variance, with the two-stage and joineR models being most biased. Despite SAS including an extra random slope term, the random intercept variance estimates were similar to R JM and Stata. Empirical standard deviations were stable across software.

Models with a flexible baseline hazard took longer to run, as would be expected. Stata were exceptionally slow in this scenario. Comparing the models using NA (nonadaptive) quadrature (e.g. R JM PWC NA) to the corresponding models using pseudo-adaptive quadrature (e.g. R JM PWC) shows that the nonadaptive versions slightly increased the runtime but otherwise choice of numerical integration algorithm made very little difference in the results. 
Table 6. Coverage probabilities for Scenario 1 (in \%).

\begin{tabular}{lcccccccc}
\hline Software & n models & Intercept & Time & X1 & X2 & Surv X1 & Surv X2 & Assoc. \\
\hline Two-stage & 100 & 88 & 94 & 93 & 94 & $\mathbf{9 7}$ & 91 & 93 \\
R JM Weib & 93 & 86 & 91 & 92 & $\mathbf{9 6}$ & $\mathbf{9 7}$ & 90 & $\mathbf{9 8}$ \\
R JM Weib NA & 92 & 86 & 91 & 91 & $\mathbf{9 7}$ & $\mathbf{9 7}$ & 91 & $\mathbf{9 8}$ \\
R JM PWC & 100 & 87 & 92 & 93 & $\mathbf{9 6}$ & $\mathbf{9 7}$ & 91 & $\mathbf{9 7}$ \\
R JM PWC NA & 100 & 86 & 92 & 92 & $\mathbf{9 7}$ & $\mathbf{9 7}$ & 91 & $\mathbf{9 7}$ \\
R JM Spl & 93 & 86 & 91 & 92 & $\mathbf{9 6}$ & $\mathbf{9 7}$ & 90 & $\mathbf{9 7}$ \\
R JM Spl NA & 93 & 85 & 91 & 91 & $\mathbf{9 7}$ & $\mathbf{9 7}$ & 90 & $\mathbf{9 7}$ \\
R JM Unspec NA & 100 & 86 & 92 & 92 & $\mathbf{9 6}$ & 92 & 91 & 79 \\
SAS \% JM Weib & 100 & 87 & 94 & 93 & $\mathbf{9 6}$ & $\mathbf{9 7}$ & 91 & $\mathbf{9 7}$ \\
SAS \%JM PWC & 100 & 83 & 91 & 93 & $\mathbf{9 6}$ & 94 & 89 & 92 \\
Stata Weib & 33 & 58 & 85 & 82 & 88 & $\mathbf{9 7}$ & 91 & $\mathbf{9 7}$ \\
R joineR & 100 & 85 & 94 & 92 & 92 & $\mathbf{9 9}$ & $\mathbf{9 6}$ & 89 \\
\hline
\end{tabular}

*The SAS longitudinal model is misspecified, including a random slope not in the data generation model.

${ }^{* *}$ The Surv X1 and Surv X2 estimates are compared to the value to which they converge and standard errors are estimated through bootstrapping. Details in Section S2.

Table 7. Average runtime for Scenarios 1, 2 and 3.

\begin{tabular}{lccr}
\hline & \multicolumn{3}{c}{ Average run times (seconds) } \\
\cline { 2 - 4 } Software & Scenario 1 & Scenario 2 & Scenario 3 \\
\hline Two-stage & 0.5 & 1.5 & 0.1 \\
R JM Weib & 3.1 & 10.9 & 5.6 \\
R JM Weib NA & 5.3 & 44.5 & 8.2 \\
R JM PWC & 7.8 & 20.6 & 11.7 \\
R JM PWC NA & 10.5 & 85.2 & 15.8 \\
R JM Spl & 21.1 & 28.5 & 15.4 \\
R JM Spl NA & 23.7 & 104.9 & 20.6 \\
R JM Unspec NA & 6.6 & 223.8 & 93.7 \\
SAS \%JM Weib & 35.6 & 49.7 & 10.6 \\
SAS \%JM PWC & 94.5 & 392.5 & 49.3 \\
Stata Weib & 1201.3 & 609.6 & 661.9 \\
R joineR & 96.9 & 121.1 & 48.5 \\
\hline
\end{tabular}

${ }^{*}$ JoineR run times include estimating bootstrapped standard errors with 50 bootstrapped samples.

\subsection{Scenario 2}

Our Scenario 2 includes a random intercept and slope joint model, sometimes called the random coefficients model, generated from the following equations.

$$
\begin{aligned}
Y_{i j}=m_{i}\left(t_{i j}\right)+e_{i j} & =\beta_{0}+\beta_{1} t_{i j}+\beta_{2} X_{1 i}+\beta_{3} X_{2 i}+b_{0 i}+b_{1 i} t_{i j}+e_{i j} . \\
\lambda_{i}(t) & =\exp \left(\gamma_{0}+\gamma_{1} X_{1 i}+\gamma_{2} X_{2 i}+\alpha m_{i}(t)\right) .
\end{aligned}
$$

As in Scenario 1, there are two covariates, one binary and one continuous. The random effects are bivariate normal. The parameter values used are shown thereafter.

$$
\begin{array}{lll}
X_{1 i} \sim \text { Bernoulli(0.5) }, & & \\
X_{2, i} \sim N(0,1), & \beta_{0}=1 & \gamma_{0}=-4.4 \\
\left(t_{i 1}, t_{i 2}, \ldots, t_{i 7}\right)=(0,0.5,1,1.5,2,2.5,3), & \beta_{1}=0.2 & \gamma_{1}=0.1 \\
e_{i j} \sim N(0,4) & \beta_{2}=-0.1 & \gamma_{2}=0.25 \\
b_{i}=\left(b_{0 i}, b_{1 i}\right)^{T} \sim N\left(\left(\begin{array}{c}
0 \\
0
\end{array}\right),\left(\begin{array}{cc}
1 & 0.5 \\
0.5 & 1
\end{array}\right)\right) & \beta_{3}=0.4 & \alpha=0.5
\end{array}
$$


(a) Longitudinal Intercept

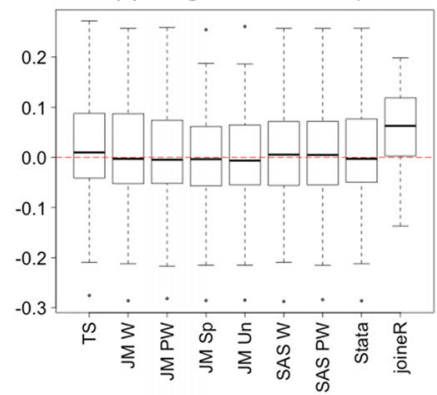

(d) Longitudinal X2

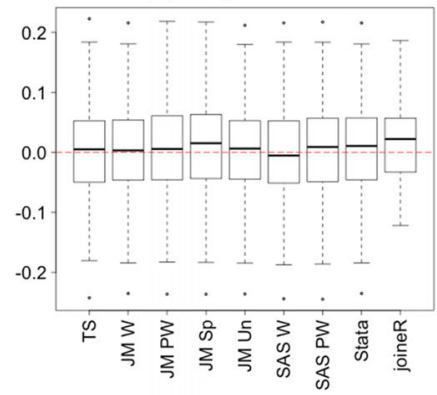

(g) Associations

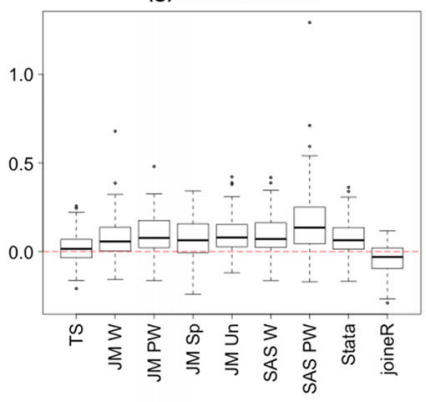

(j) Random Effects Covariance

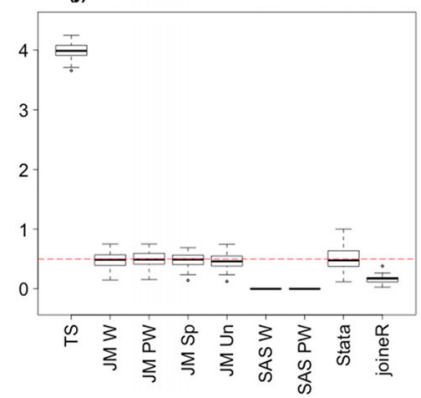

(b) Longitudinal Time

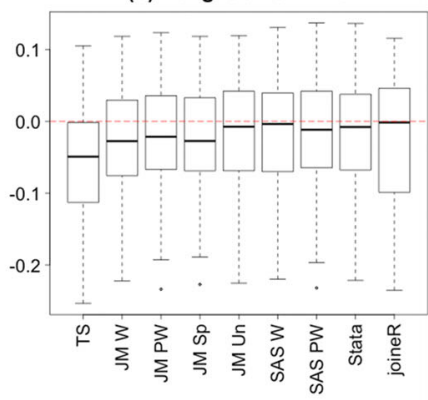

(e) Survival $\times 1$

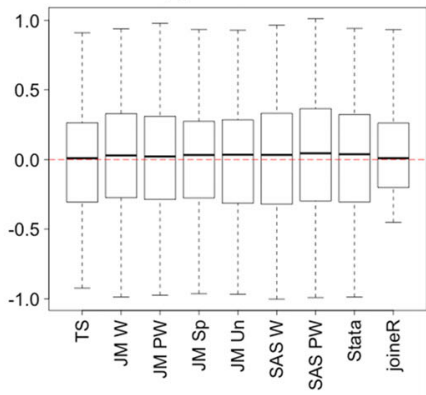

(h) Random Intercept Variance

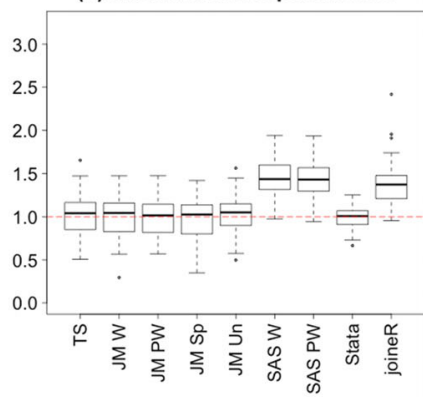

(c) Longitudinal X1

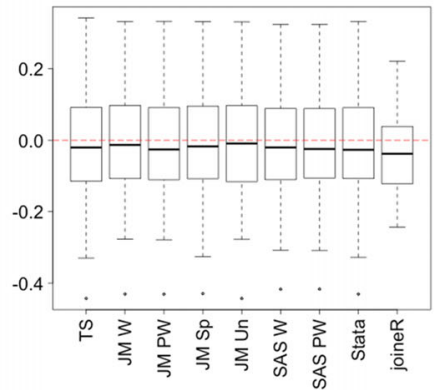

(f) Survival X2

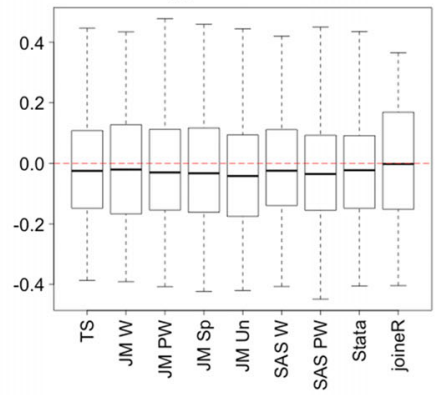

(i) Random Slope Variance

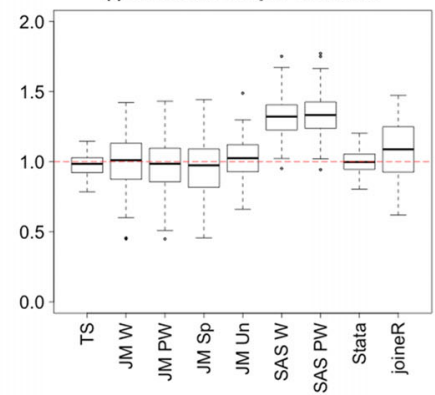

Figure 2. Boxplots showing the bias of the Scenario 2 estimates. Model names abbreviated as: TS $=$ TwoStage, JM W=R JM Weibull, $J M P W=R$ JM Piece-Wise, JM Sp =R JM Spline, JM Un =R JM Unspeci?ed NA, SAS W=SAS Weibull, SAS PW $=S A S$ Piece-Wise. Figures $2(h, i, j)$ plot the actual values estimated for the random effect variances and covariance with the dotted line at the true value. [Colour figure can be viewed at wileyonlinelibrary.com] 
Table 8. Coverage probabilities for Scenario 2 (in \%).

\begin{tabular}{lcccccccc}
\hline Software & n models & Intercept & Time & X1 & X2 & Surv X1 & Surv X2 & Assoc. \\
\hline Two-stage & 100 & $\mathbf{9 8}$ & 87 & $\mathbf{9 7}$ & 89 & $\mathbf{9 5}$ & 94 & $\mathbf{9 7}$ \\
R JM Weib & 88 & $\mathbf{9 5}$ & 93 & $\mathbf{9 7}$ & 91 & 93 & 90 & 90 \\
R JM Weib NA & 95 & $\mathbf{9 8}$ & $\mathbf{9 6}$ & $\mathbf{9 8}$ & 91 & 94 & 93 & 92 \\
R JM PWC & 87 & $\mathbf{9 7}$ & 94 & $\mathbf{9 8}$ & 90 & $\mathbf{9 5}$ & 92 & $\mathbf{9 8}$ \\
R JM PWC NA & 94 & $\mathbf{9 7}$ & $\mathbf{9 6}$ & $\mathbf{9 8}$ & 91 & $\mathbf{9 6}$ & 91 & $\mathbf{9 5}$ \\
R JM Spl & 83 & $\mathbf{9 5}$ & 93 & $\mathbf{9 5}$ & 87 & 93 & 92 & $\mathbf{9 5}$ \\
R JM Spl NA & 94 & $\mathbf{9 7}$ & $\mathbf{9 6}$ & $\mathbf{9 8}$ & 91 & $\mathbf{9 5}$ & 93 & $\mathbf{9 6}$ \\
R JM Unspec NA & 85 & $\mathbf{9 6}$ & $\mathbf{9 5}$ & $\mathbf{9 8}$ & 92 & 91 & 92 & 64 \\
SAS \%JM Weib & 100 & 92 & 91 & 91 & 86 & 88 & 89 & 86 \\
SAS \%JM PWC & 100 & $\mathbf{9 8}$ & $\mathbf{9 7}$ & $\mathbf{9 7}$ & 91 & $\mathbf{9 5}$ & 92 & 89 \\
Stata Weib & 99 & $\mathbf{9 7}$ & $\mathbf{9 7}$ & $\mathbf{9 7}$ & 90 & 94 & 93 & 90 \\
R joineR & 25 & $\mathbf{1 0 0}$ & 88 & $\mathbf{1 0 0}$ & $\mathbf{9 6}$ & $\mathbf{9 6}$ & 80 & 92 \\
\hline
\end{tabular}

${ }^{*}$ The Surv X1 and Surv X2 estimates are compared to the value to which they converge and standard errors are estimated through bootstrapping. Details in Section S2.

We draw independent censoring times $C_{i} \sim \operatorname{Unif}(1.25,3.25)$ and the observed times are $T_{i}=\min \left(T_{i}^{*}, C_{i}\right)$. There was approximately $10 \%$ censoring.

Each software, JM, joineR in R, \%JM in SAS and stjm in Stata, fits (12) to the longitudinal part of the data. The default covariance structure was used in all models. As in Scenario 1 , several survival submodels were evaluated. The R JM package, SAS and Stata fit a currentvalue survival submodel as in (13). The shared-parameter model thereafter was fit with joineR which is a reparameterisation of the current-value model similar to Scenario 1.

$$
\lambda_{i}(t)=\lambda_{0}(t) \exp \left(\gamma_{1}^{*} X_{1 i}+\gamma_{1}^{*} X_{2 i}+\alpha\left(b_{0 i}+b_{1 i} t\right)\right)
$$

The baseline hazards are the same as in (11) including models using both adaptive and nonadaptive (NA) in R JM Weib, PWC and Spl. Default settings were used for the piecewise constant and spline functions. Finally, a two-stage model was fit for comparison.

Results for Scenario 2 are in Figure 2, and Table 8. Table 7 shows the run times for this scenario. More results including bias, MSE and confidence interval widths are included in Supporting Information.

The software were able to fit models to most of the 100 simulated datasets except for joineR. The joineR models could fit only around a quarter of the datasets. Coverage probabilities were similar for all software with one exception. Coverage for the association parameter was noticeably lower for R JM Unspec NA. The confidence interval widths were generally very similar in each software. As in Scenario 1, on average, the confidence intervals around the association estimate in R JM Unspec NA were considerably tighter.

In the boxplots, we see that all R JM models and Stata estimated the random effect covariance parameters well. In SAS, the covariance of the random effects was assumed zero according to the macro defaults, and we see that the variance of the intercept and the slope were overestimated by SAS. The two-stage model estimated the random effect variances well but severely overestimated the covariance, possibly including additional variance in the longitudinal submodel random effects estimates that would be explained by the association with the survival submodel in a joint model. Empirical standard deviations were consistent in all software. We see that the random coefficients models in this scenario took longer to run on average than the models in Scenario 1 for all software except Stata. Using nonadaptive quadrature increased runtime more compared to Scenario 1. 


\subsection{Scenario 3}

The final simulation scenario utilised a shared coefficients form as in (3), with a random intercept only. The data were generated from

$$
\begin{gathered}
Y_{i j}=\beta_{0}+\beta_{1} t_{i j}+\beta_{2} X_{1 i}+\beta_{3} X_{2 i}+b_{0 i}+e_{i j} . \\
\lambda_{i}(t)=\exp \left(\gamma_{0}+\gamma_{1} X_{1 i}+\gamma_{2} X_{2 i}+\alpha b_{i}\right) .
\end{gathered}
$$

Parameter values are thereafter.

$$
\begin{array}{lll}
X_{1 i} \sim \text { Bernoulli(0.5), } & \beta_{0}=6 & \gamma_{0}=-4.4 \\
X_{2, i} \sim N(0,0.5), & \beta_{1}=0.2 & \gamma_{1}=0.9 \\
\left(t_{i 1}, t_{i 2}, \ldots, t_{i 7}\right)=(0,0.5,1,1.5,2,2.5,3), & \beta_{2}=-0.1 & \gamma_{2}=1.2 \\
e_{i j} \sim N(0,4), & \beta_{3}=0.4 & \alpha=0.5 \\
b_{i} \sim N(0,0.7) & &
\end{array}
$$

For this scenario, independent censoring times were drawn $C_{i} \sim \operatorname{Unif}(1.25,3.25)$. There was approximately $20 \%$ censoring.

A random intercept only shared parameter survival submodel was used since the most software could fit the correct model. The R function joineR and Stata fit the same longitudinal submodel in (14). Again, the default in SAS is to fit a longitudinal model with both a random intercept and slope as was the case in Scenario 1 which in this case is an misspecified model. The joineR, SAS and Stata software fit (15) to the survival part. The JM package in R only fits joint models with a current-value association, which amounts to a reparameterisation of the shared parameter models. In this scenario, R JM fits the survival submodel in (16). The survival coefficients will converge to a combination of true parameters, namely, $\gamma_{1}^{*}=\gamma_{1}-\alpha \beta_{2}$ and $\gamma_{2}^{*}=\gamma_{2}-\alpha \beta_{3}$. See Section S2 for details.

$$
\lambda_{i}(t)=\lambda_{0}(t) \exp \left(\gamma_{1}^{*} X_{1 i}+\gamma_{2}^{*} X_{2 i}+\alpha m_{i}(t)\right) .
$$

Baseline hazards are listed in (11). Both adaptive and nonadaptive (NA) numerical integration was again utilised in $\mathrm{R}$ JM with Weibull, piecewise constant and spline baseline hazard functions. We used the default settings to create piecewise constant and spline baseline hazard functions. A two-stage model was also fit with the survival model being a standard semi-parametric Cox model.

Figure 3 and Table S3 show the results for Scenario 3. Coverage probabilities are shown in Tables 9 and 7 which show the run times for all scenarios. Additional results are included in Supporting Information.

Models could be fit to all or almost all of the 100 simulated datasets by each software in this scenario. $\mathrm{R}$ joineR had a considerably lower coverage probability for the $X_{2}$ coefficient in the survival submodel. Nearly all software except R JM Weib (and R JM Weib NA) had relatively low coverage for the association parameter, with the coverage from R JM Unspec NA being especially poor. Again, the average width of confidence intervals around the association estimate from R JM Unspec NA was much smaller than all other software. The average confidence interval widths for $X_{1}$ and $X_{2}$ from R JM Spl are much larger than the other software, possibly due to poor model fitting on the simulated datasets corresponding to the outliers.

$\mathrm{R}$ JM and Stata estimated the random intercept variance best although all (except two-stage) underestimated this value. The two-stage model severely overestimated this value as in the first two scenarios. Interestingly fitting models to this data with R JM Spl produced estimates with larger bias for most covariates and considerably smaller MSE for some covariates compared 
(a) Longitudinal Intercept

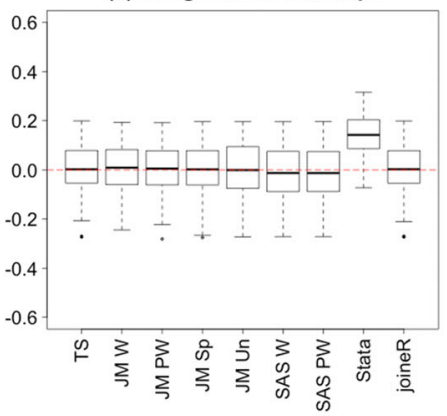

(d) Longitudinal X2

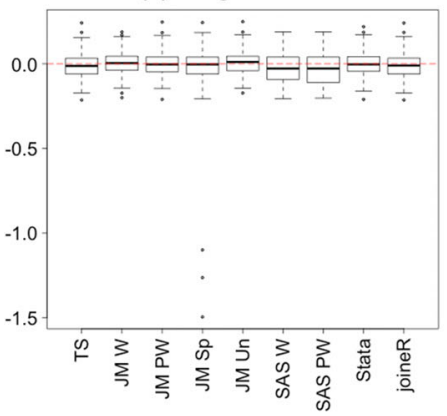

(g) Associations

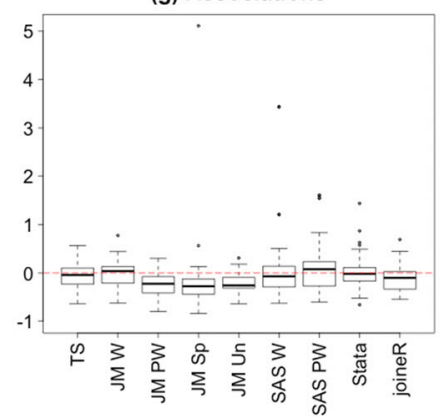

(b) Longitudinal Time

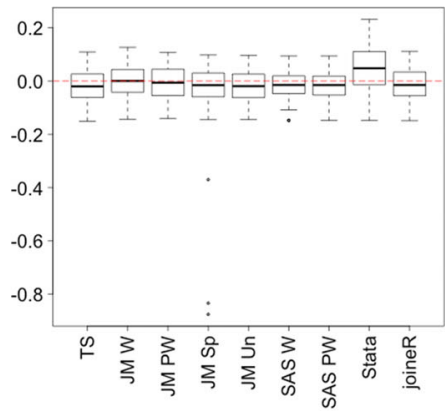

(e) Survival X1

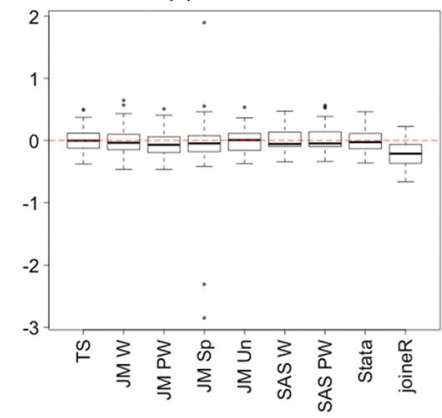

(h) Random Effects Variance

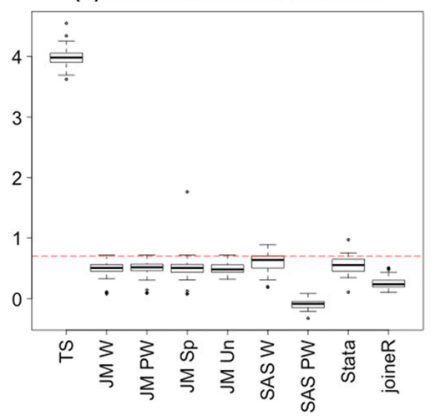

(c) Longitudinal X1

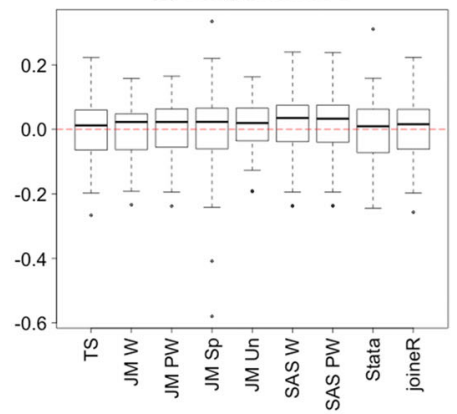

(f) Survival X2

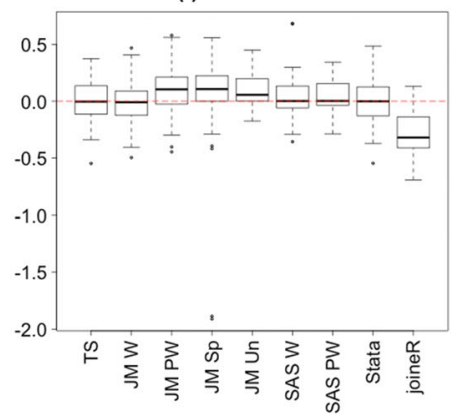

Figure 3. Boxplots showing the bias of the Scenario 3 estimates. Model names abbreviated as: TS=TwoStage, JMW $=R J M$ Weibull, JM $P W=R$ JM Piece-Wise, JM Sp=R JM Spline, JM Un=R JM Unspecified NA, SAS W=SAS Weibull, SAS PW= SAS Piece-Wise. Figure 3(h) plots the actual values estimated for the random effect variance with the dotted line at the true value. Note: Outliers in Intercept and Association from JM Sp have been omitted to aid in interpreting the boxplots. [Colour figure can be viewed at wileyonlinelibrary.com]

to R JM with the other baseline options. We noted that SAS produced survival submodel $X_{2}$ coefficient estimates with an unusually large MSE. Also, joineR generated more biased estimates for the survival submodel despite fitting a model in the same form as the data generating model. As in the first two scenarios, empirical standard deviations were very similar across all software. Lastly, SAS and R joineR ran for notably shorter times than in the first two scenarios.

\section{Bayesian Models and Associated Software}

Joint modelling from a Bayesian perspective has also been implemented. The full posterior distribution conforms to the structure 
Table 9. Coverage probabilities for Scenario 3 (in \%).

\begin{tabular}{lcccccccc}
\hline Software & n models & Intercept & Time & X1 & X2 & Surv X1 & Surv X2 & Assoc. \\
\hline Two-stage & 100 & 91 & 94 & $\mathbf{9 8}$ & 93 & $\mathbf{9 7}$ & 92 & 94 \\
R JM Weib & 82 & $\mathbf{9 5}$ & 93 & $\mathbf{9 9}$ & 91 & 88 & $\mathbf{9 5}$ & $\mathbf{9 5}$ \\
R JM Weib NA & 80 & 91 & 90 & $\mathbf{9 9}$ & 91 & $\mathbf{9 6}$ & 94 & $\mathbf{9 6}$ \\
R JM PWC & 88 & $\mathbf{9 5}$ & 93 & $\mathbf{9 9}$ & 93 & $\mathbf{9 5}$ & $\mathbf{9 8}$ & 82 \\
R JM PWC & 89 & 94 & 93 & $\mathbf{9 8}$ & 92 & $\mathbf{9 5}$ & $\mathbf{9 7}$ & 80 \\
R JM Spl & 97 & 94 & $\mathbf{9 5}$ & $\mathbf{9 8}$ & 92 & $\mathbf{9 7}$ & $\mathbf{9 5}$ & 87 \\
R JM Spl NA & 100 & 94 & 94 & $\mathbf{9 8}$ & 92 & $\mathbf{9 7}$ & $\mathbf{9 5}$ & 88 \\
R JM Unspec NA & 45 & 93 & 89 & $\mathbf{1 0 0}$ & 89 & 93 & 88 & 11 \\
SAS \%JM Weib & 100 & 87 & $\mathbf{9 6}$ & $\mathbf{9 6}$ & 94 & $\mathbf{1 0 0}$ & $\mathbf{9 7}$ & 84 \\
SAS \%JM PWC & 100 & 88 & $\mathbf{9 6}$ & $\mathbf{9 6}$ & 94 & $\mathbf{9 8}$ & $\mathbf{1 0 0}$ & 80 \\
Stata Weib & 89 & 51 & 85 & $\mathbf{9 8}$ & 91 & $\mathbf{9 9}$ & $\mathbf{1 0 0}$ & 80 \\
R joineR & 100 & 93 & 94 & $\mathbf{9 5}$ & 89 & 80 & 51 & 91 \\
\hline
\end{tabular}

${ }^{*}$ The SAS longitudinal model is misspecified, including a random slope not in the data generation model.

${ }^{* *}$ The Surv X1 and Surv X2 estimates are compared to the value to which they converge and standard errors are estimated through bootstrapping. Details in Section S2.

$$
f(\theta, b \mid T, \delta, y) \propto \prod_{i=1}^{n}\left(f\left(y_{i} \mid b_{i} ; \theta\right) f\left(T_{i}, \delta_{i} \mid b_{i} ; \theta\right) f\left(b_{i} ; \theta\right)\right) \pi(\theta),
$$

where $\pi(\theta)$ denotes the joint prior for the model parameters. Markov Chain Monte Carlo (MCMC) techniques such as Gibbs sampling or Metropolis-Hastings algorithm can be used for inference (Bekele \& Shen, 2005; Faucett \& Thomas, 1996; Gould et al., 2014; Henderson et al., 2000; Rizopoulos \& Ghosh, 2011; Tsiatis \& Davidian, 2004; Yu et al., 2004). The Bayesian implementation was not compared to the simulations for the maximum likelihood methods in the last section. Instead, in this section, we will discuss the single option for Bayesian joint modelling in the software and also use this to analyse the adrenal cancer data.

\subsection{JMbayes Package in $R$}

The JMbayes package in $\mathrm{R}$ was written to fit joint models in a Bayesian framework (Rizopoulos, 2016c). The function for fitting joint models is named jointModelBayes (). The arguments for this function are very similar to the jointModel () function in the JM package in R. A linear mixed model is fit to the longitudinal data unless the user specifies a different distribution with the desLong argument. A relative risk model is fit for the timeto-event data. The baseline hazard is estimated using splines, either penalised P-splines (the default) or regression splines (Rizopoulos, 2016b). The param argument determines the form of the association between the submodels. The default association is current value as in (2). Other options are association based on the current slope of the longitudinal trajectory analogous to the R JM package in (7), both the current value and slope, or shared parameters like in (3). A final association option is a combination of shared random effects and fixed effects, $\beta^{*}$, such as $\lambda_{i}\left(t \mid M_{i}(t)\right)=\lambda_{0}(t) \exp \left\{X_{2 i} \gamma+\alpha\left(\beta^{*}+b_{i}\right)\right\}$. Available functionalities in the JMbayes package are listed in Table 5. Extra flexibility is available for the association structure since the user can define any transformation function using the extraForm and transFun arguments. Using this, the association can be defined to be any function of the current value or any function of the shared random effects.

Estimation is performed using MCMC sampling from the posterior conditional distributions of the random effects and the parameters. Usually, a random walk Metropolis can be 
Table 10. Parameter estimates and credible intervals for the joint models fit to the adrenal data with JMbayes.

\begin{tabular}{ll}
\hline Variable & \multicolumn{1}{c}{ Estimate } \\
\hline Intercept & $62.0(57.4,66.5)$ \\
Time & $-0.5(-0.9,-0.2)$ \\
Age & $-0.2(-0.24,-0.07)$ \\
Stages 3 or 4 & $-0.4(-1.4,0.5)$ \\
Tumour grade: high & $-0.5(-3.2,2.2)$ \\
Tumour grade: unknown & $-3.2(-6.7,0.4)$ \\
Surv-age & $-0.007(-0.02,0.01)$ \\
Surv-stage 3 or 4 & $0.2(0.01,0.3)$ \\
Surv-tumour grade: high & $0.40(-0.03,0.9)$ \\
Surv-tumour grade: unknown & $0.8(0.2,1.4)$ \\
Association & $-0.09(-0.1,-0.06)$ \\
\hline
\end{tabular}

used, but in some cases, Metropolis-Hastings or slice sampling are needed (Rizopoulos, 2016c). Initial values can be set using the init argument, but if left unspecified, initial values are taken from the outputs from the separate models that are included as arguments to the jointModelBayes () function. Priors can also be specified by the user with the priors argument. If not specified, standard prior distributions are used: all the fixed parameters from both submodels as well as the association parameter are given independent diffuse normal priors, a inverse Wishart prior is assumed for the covariance matrix of the random effects when fitting a normally distributed longitudinal outcome, and an inverse Gamma prior for the error variance (Rizopoulos, 2016c). The JMbayes package also includes functions for plotting and running dynamic predictions (Rizopoulos, 2016b).

\subsection{Data Analysis}

A joint model with the current value association as in (4) and (5) with a P-spline baseline hazard is fit to the adrenal cancer data from Section 3 using JMbayes. MCMC is run for 52,000 iterations with a burn-in of 2000. The results are shown in Table 10. The JMbayes estimates are generally similar to the frequentist current-value results in Table 2, and interpretations of the covariate effects on psoas density and survival are the same.

\section{Extensions and Specialised Joint Models and their Implementation}

The growing interest in joint modelling has led to many extensions, such as joint modelling with competing risks, recurrent events or multiple longitudinal processes. The addition of many software to fit standard joint models has also encouraged the development of more specialised software. Here, we briefly describe several model extensions and software available for implementation. See Section S4 for more implementations via software. The extent of software implementation for specialised joint modelling is likely to increase in the future.

Up until this point, we have focused on longitudinal measurements with a Gaussian distribution. Yet situations often arise that require a non-Gaussian outcome in the longitudinal submodel, such as a logistic or Poisson model. The JM and JMbayes software in R as well as the \%JM macro in SAS, described in Section 4, can accommodate non-Gaussian longitudinal processes. The Joint $1 \mathrm{cmm}$ () function in the $1 \mathrm{cmm}$ package in $\mathrm{R}$ can implement different distributions in the longitudinal submodel of a latent class mixed model (Proust-Lima et al., 2016). 
Joint latent class mixed modelling is an extension that can be used to investigate class-specific differences. These models typically include three submodels, a multinomial logistic model to determine the latent class, a class-specific linear (or latent process) mixed model and a classspecific survival model. The number of latent classes must be set a priori based on knowledge of the situation from which the data were collected. The $1 \mathrm{cmm}$ package in $\mathrm{R}$ was written for latent class mixed modelling and includes a function, Joint $1 \mathrm{cmm}()$, which fits a joint latent class mixed model for longitudinal and time-to-event data (Proust-Lima et al., 2016). The baseline risk can be common or class specific. Choice of initial values is important, and it is preferred that the user specify initial values with the B argument over using the defaults.

In medical studies, it is likely more than one biomarker is measured for each patient. This necessitates joint models with multiple longitudinal variables. Hickey et al. (2016) review developments in multivariate joint models including software implementations. Hickey et al. mentioned a new package for multivariate joint models, sjmsoft for $\mathrm{R}$, available from the author's website (Brown, 2005). Another option is the R package joineRML (Hickey et al., 2018).

An interesting but currently less studied extension is joint modelling with competing risks. The longitudinal submodel for a single longitudinal outcome is the same as a single risk joint model. Literature has almost exclusively focused on a survival submodel with proportional cause-specific hazards (Armero et al., 2016; Blanche et al., 2015; Elashoff et al., 2007; Hu et al., 2009; Huang et al., 2010; Williamson et al., 2008). Usually, the survival submodel has the following form, defining the cause specific hazard $\lambda^{(k)}$ for cause $k=1, \ldots, K$.

$$
\begin{aligned}
\lambda^{(k)}\left(t ; X_{2 i}, b_{i}, \gamma^{(k)}, \alpha^{(k)}\right) & =\lim _{h \rightarrow 0} h^{-1} P\left(t \leq T_{i}<t+h, \delta_{i}=k \mid T_{i} \geq t, X_{2 i}(t), b_{i}\right) \\
& =\lambda_{0}^{(k)}(t) \exp \left\{X_{2 i}(t) \gamma^{(k)}+\alpha^{(k)} b_{i}\right\} .
\end{aligned}
$$

The parameters are defined similar to those in Section 2.2, $X_{2 i}$ are fixed effects which have possibly cause-specific coefficients $\gamma^{(k)} ; b_{i}$ are the random effects for subject $i$ with possibly cause-specific association $\alpha^{(k)}$, and cause-specific baseline hazard $\lambda_{0}^{(k)}(t)$.

Some of the joint modelling software will fit a competing risks model. The JM package in $R$ has an option CompRisk in the jointModel () function. The joint () function in the joineR package will fit a cause-specific hazard joint model if the event indicator has multiple levels, but the documentation states this only works for two causes in addition to censoring, and no more (Philipson et al., 2012). The SAS macro \%JM can also fit a competing risk model with the option COMPETING which is listed under Additionaloptions. Documentation for the st jm Stata command states that extension to the competing risks setting is planned, but there is no indication that this has been completed (Crowther et al., 2013). There is also no built-in option for fitting a competing risks model in JMbayes. Recently, a SAS macro called $\%$ SPM was proposed specifically for fitting joint competing risks models (Wang et al., 2017). Finally, the Joint $1 \mathrm{cmm}$ ( ) function in $1 \mathrm{cmm}$ in $\mathrm{R}$ can also handle competing risks (Proust-Lima et al., 2016).

Another common situation is data including recurrent events such as repeated hospitalisations or time between system breakdown in industry. The R package frailtypack will fit a standard joint model with function long i Penal and also joint models with longitudinal measurements, a terminal event plus recurrent events using the function trivPenal (Rondeau et al., 2017; Rondeau et al., 2012).

With the increasing number of studies utilising joint modelling, it may be useful to analyse the results from multiple studies in a meta-analytic context. A package for joint modelling in a this context, joineRmeta in $\mathrm{R}$, has been developed which can pool model parameters 
from multiple joint models using standard meta-analysis techniques or analyse the data from all studies simultaneously (Sudell et al., 2018; Sudell, 2018).

\section{Concluding Remarks}

Joint modelling is a growing field of statistical research and the available software encourages the use of these complicated models in applications. We have given an overview of joint modelling methodology and then compiled a comprehensive list of available software. We compared through simulation and data analysis the most common and user-friendly software: JM, joineR and JMbayes in R, \% JM in SAS and st j $m$ in Stata. We also included a short description of extensions to joint modelling such as those that accommodate competing risks, multiple longitudinal markers or recurrent events. The \%JMfit macro developed for SAS, described in Section S4, includes some goodness-of-fit calculations such as decomposition of AIC, BIC, $\triangle \mathrm{AIC}$ and $\triangle \mathrm{BIC}$ (Zhang et al., 2016). Assessing model fit in a joint modelling framework has had limited study and further development of this area is likely in the future (Zhang et al., 2014).

Our simulations show joint modelling software is preferable to two-stage models when the longitudinal and survival processes are correlated, as the theoretical work finds. Ours is the first investigation that explored the performance of regression coefficients on both longitudinal and survival components. The packages in $\mathrm{R}$ fit only one type of association while SAS and Stata include many more options. All software we compared are similar in their performance when fitting the longitudinal submodel in simulations. There are more differences in the survival submodel options and performance. All except joineR included a flexible parametric baseline hazard which is most applicable to real data when the true model is unknown. A Cox-type survival submodel using an unspecified baseline hazard is currently available in the $R$ packages joineR and JM. In each of our simulation scenarios, we found that the average width of the confidence intervals around the association estimate were smaller than all other software. This could be evidence of underestimation of standard error. This is similar to what was found in simulations in Yuen and Mackinnon (2016). Otherwise, we did not see underestimation for the other covariates. Still, documentation of the R JM package does not indicate that any techniques are used to correct for the theoretical underestimation. Hence, this should be considered when using the $\mathrm{R}$ JM package with a Cox type survival submodel. A recently developed R package JSM fits semiparametric joint models while estimating standard errors with numerical differentiation in an EM algorithm as described by Xu et al. $(2014,2018)$. We found convergence issues when using the adaptive Gauss-Hermite quadrature option only when using an unspecified baseline hazard in the R JM function. Additionally, we saw in our simulations that using a restrictive parametric baseline risk function that matches the truth does not aid in estimation but may shorten runtime. Unless there is a compelling reason to use a restrictive parametric function, such as Weibull, for the baseline hazard, we would recommend using a flexible hazard such as piecewise constant or spline based.

In our investigation through simulation, we found that the Stata stjm command can be very sensitive to the parameter values used in data generation. SAS can also have difficulties depending on the parameters chosen when using an unstructured covariance matrix, but the default model assuming uncorrelated random effects was always able to fit the models. The $R$ functions were always able to fit models to at least some of the datasets, no matter the parameter values used.

Basic joint modelling capabilities are available in the main statistical programming languages, R, SAS and Stata, giving the user an option to use the implementation in the language of 
their choice. Each software has good features as well as limitations. Overall, we would recommend R JM or SAS. The SAS macro \%JM offers the most functionality, including submodel, association and estimation options. $\mathrm{R}$ JM was consistently the fastest and includes almost as many options as SAS, so this may be a better option if runtime is a concern. The joine function is limited in the type of models it can fit. But if a joint model with a Gaussian longitudinal response and a shared parameter survival submodel is appropriate for the data, joineR may be a good option because of its simplicity.

Bayesian models can be very powerful and are gaining traction in joint modelling literature, but the implementation is relatively limited. Analysing joint models in a general multi-purpose Bayesian statistical programming language such as OpenBUGS or WinBUGS is possible and attractive due to its flexibility. Defining a joint model can become complicated in OpenBUGS due to the lack of closed form for the integrals encountered in estimation. A common simplifying assumption is that the survival process follows a parametric distribution, often Weibull (Guo, 2003; Guo \& Carlin, 2004). Full proportional hazards survival submodels have been implemented in the literature, and code is available in Supporting Information of Rizopoulos and Ghosh (2011) for the case of multiple longitudinal outcomes and Andrinopouslou et al. (2013) for the case of two longitudinal variables and competing risks data. A competing risks joint model has been implemented in WinBUGS (Deslandes \& Chevret, 2010).

Our investigation was focused on giving an overview of the available software and their features. Our simulations were limited to three scenarios that could be modelled in each common software. Other simulation scenarios were explored but not every software was able to fit models to the data. Running more simulations with different data forms and more focus on time slopes in longitudinal models, different forms of the submodels and various associations would add to the knowledge of these software. Further development of this group of software is expected based on the continued research in this field and the applicability of these models. With increased generalisation in the form of each part (longitudinal and survival submodels, and the association), the software could accommodate many more types of data. Some software are currently being developed and released to accommodate useful extensions such as competing risks and multiple longitudinal outcomes as we discussed. Further work on more robust estimation as well as faster algorithms would be useful. Fitting a simple joint model with only a few covariates is not prohibitively restrictive on time. The time required will increase with an increase in the number of covariates or a non-normal longitudinal models, especially if one has to rely on re-sampling techniques for inference purposes. Such extensions may require creative enhancements and approximations that would be computationally efficient. An important area in joint modelling that is largely unexplored is model diagnostics. While some of the software offer some basic diagnostics (Rizopoulos, 2010; 2016c) and dynamic predictions (Crowther et al., 2013; Garcia-Hernandez \& Rizopoulos, 2015; Rizopoulos, 2010; 2016c), there is room for further expansion.

\section{Acknowledgement}

This work was partially supported by grant CA 083654 from the US National Institutes of Health.

\section{Supporting Information}

Additional Supporting Information may be found online in the supporting information tab for this article. 


\section{References}

Andrinopouslou, E.-R., Rizopoulos, D., Takkenberg, J. J. \& Lesaffre, E. (2013). Joint modeling of two longitudinal outcomes and competing risk data. Stat. Med., 33, 3167-3178.

Armero, C., Forte, A., Perpiñán, H., Sanahuja, M. J. \& Agustí, S. (2016). Bayesian joint modeling for assessing the progression of chronic kidney disease in children. Stat. Methods Med. Res., 27, 1-17.

Bekele, B. N. \& Shen, Y. (2005). A Bayesian approach to jointly modeling toxicity and biomarker expression in a phase I/II dose-finding trial. Biometrics, 61, 344-354.

Blanche, P., Proust-Lima, C., Loubère, L., Berr, C., Dartigues, J.-F. \& Jacqmin-Gadda, H. (2015). Quantifying and comparing dynamic predictive accuracy of joint models for longitudinal marker and time-to-event in presence of censoring and competing risks. Biometrics, 71, 102-113.

Brown, E. R. (2005). Software. http://faculty.washington.edu/elizab/software.html, Accessed: 2018-02-05.

Brown, E. R. \& Ibrahim, J. G. (2003). A Bayesian semiparametric joint hierarchical model for longitudinal and survival data. Biometrics, 59(2), 221-228.

Crowther, M. J., Abrams, K. R. \& Lambert, P. C. (2013). Joint modeling of longitudinal and survival data. Stata J., 13(1), 165-184.

De Gruttola, V. \& Tu, X. M. (1994). Modelling progression of CD4-lymphocyte count and its relationship to survival time. Biometrics, 50(4), 1003.

Deslandes, E. \& Chevret, S. (2010). Joint modeling of multivariate longitudinal data and the dropout process in a competing risk setting: Application to ICU data. Medical Research Methodology, 10(69), 1-13.

Efron, B. \& Tibshirani, R. J. (1993). An Introduction to the Bootstrap Boca Raton, Florida: Chapman \& Hall. pages 184-187.

Elashoff, R. M., Li, G. \& Li, N. (2007). An approach to joint analysis of longitudinal measurements and competing risks failure time data. Stat. Med., 26(14), 2813-2835.

Faucett, C. L. \& Thomas, D. C. (1996). Simultaneously modelling censored survival data and repeatedly measured covariates: A Gibbs sampling approach. Stat. Med., 15(15), 1663-1685.

Garcia-Hernandez, A. \& Rizopoulos, D. (2015). \%JM Macro Reference Manual. http://www.jm-macro.com/uploads/ 2/6/0/6/26064877/jmmacrov1.07_referencemanual.pdf, Online; accessed December 1, 2015.

Gould, A. L., Boye, M. E., Crowther, M. J., Ibrahim, J. G., Quartey, G., Micallef, S. \& Bois, F. Y. (2014). Joint modeling of survival and longitudinal non-survival data: Current methods and issues. Report of the DIA Bayesian joint modeling working group. Stat. Med., 34(14), 2181-2195.

Guo, X. (2003). WinBUGS 1.4 code for joint model (Model XI in Guo and Carlin paper). http://www.biostat.umn. edu/ brad/software/ModelXInew.bug, Online; accessed December 1, 2015.

Guo, X. \& Carlin, B. P. (2004). Separate and joint modeling of longitudinal and event time data using standard computer packages. Amer. Stat., 58(1), 16-24.

Henderson, R., Diggle, P. \& Dobson, A. (2000). Joint modelling of longitudinal measurements and event time data. Biostatistics, 1(4), 465-480.

Hickey, G. L., Philipson, P. \& Jorgensen, A. (2018). Package 'joineRML': Joint modeling of multivariate longitudinal data and time-to-event outcomes. https://cran.r-project.org/web/packages/joineRML/joineRML.pdf, Accessed: 2018-02-05.

Hickey, G. L., Philipson, P., Jorgensen, A. \& Kolamunnage-Dona, R. (2016). Joint modelling of time-to-event and multivariate longitudinal outcomes: Recent developments and issues. BMC Medical Research Methodology, 16(117), $1-15$.

Holcombe, S., Enchakalody, B., Zhang, P. \& Wang, S. (2016). Morphomics Data Dictionary. http://www.med.umich. edu/surgery/morphomics/data_dictionary, Accessed: 2017-08-28.

Hseih, F., Tseng, Y.-K. \& Wang, J.-L. (2006). Joint modeling of survival and longitudinal data: A likelihood approach revisited. Biometrics, 62, 1037-1043.

Hu, W., Li, G. \& Li, N. (2009). A Bayesian approach to joint analysis of longitudinal measurements and competing risks failure time data. Stat. Med., 28(11), 1601-1619.

Huang, Y., Dagne, G. \& Wu, L. (2011). Bayesian inference on joint models of HIV dynamics for time-to-event and longitudinal data with skewness and covariate measurement errors. Stat. Med., 30(24), 2930-2946.

Huang, X., Li, G., Elashoff, R. M. \& Pan, J. (2010). A general joint model for longitudinal measurements and competing risks survival data with heterogeneous random effects. Lifetime Data Anal., 17(1), 80-100.

Huang, X., Stefanski, L. A. \& Davidian, M. (2009). Latent-model robustness in joint models for a primary endpoint and a longitudinal process. Biometrics, 65(3), 719-727.

Ibrahim, J. G., Chu, H. \& Chen, L. M. (2010). Basic concepts and methods for joint models of longitudinal and survival data. J. Clin. Oncol., 28(16), 2796-2801.

Lehmann, A. (2009). Joint modeling of degradation and failure time data. J. Stat. Plan. Infer., 139(5), 1693-1706. 
Li, E., Wang, N. \& Wang, N.-Y. (2007). Joint models for a primary endpoint and multiple longitudinal covariate processes. Biometrics, 63(4), 1068-1078.

Liu, L. \& Huang, X. (2009). Joint analysis of correlated repeated measures and recurrent events processes in the presence of death, with application to a study on acquired immune deficiency syndrome. J. R. Stat. Soc.: Ser. C: Appl. Stat., 58(1), 65-81.

Mccrink, L. M., Marshall, A. H. \& Cairns, K. J. (2013). Advances in joint modelling: A review of recent developments with application to the survival of end stage renal disease patients. Int. Stat. Rev., 81(2), 249-269.

Miller, B. S., Ignatoski, K. M., Daignault, S., Doherty, C. L. M., Gauger, P. G., Hammer, G. D., Wang, S. C., Doherty, G. M. \& T. U of Michigan Analytical Morphomics Group. (2012). Worsening central sarcopenia and increasing intra-abdominal fat correlate with decreased survival in patients with adrenocortical carcinoma. World J. Surg., 36, 1509-1516.

Murphy, S. A. \& van der Vaart, A. W. (2000). On profile likelihood. J. Am. Stat. Assoc., 95(450), 449-465.

Philipson, P., Sousa, I., Diggle, P., Williamson, P., Kolamunnage-Dona, R. \& Henderson, R. (2012). Package 'joineR': Joint modeling of repeated measurements and time-to-event data. https://cran.r-project.org/web/packages/joineR/ joineR.pdf, Accessed: 2016-10-12.

Pinheiro, J., Bates, D., DebRoy, S., Sarkar, D., EISPACK, Heisterkamp, S. \& Van Willigen, B. (2016). Package 'nlme': Linear and Nonlinear Mixed Effects Models. https://cran.r-project.org/web/packages/nlme/nlme.pdf, Accessed: 2016-10-12.

Proust-Lima, C., Philips, V. \& Liquet, B. (2016). Estimation of Extended Mixed Models Using Latent Classes and Latent Processes: The R Package lcmm. https://arxiv.org/pdf/1503.00890.pdf, Online; accessed November 1, 2016.

Proust-Lima, C. \& Taylor, J. M. G. (2009). Development and validation of a dynamic prognostic tool for prostate cancer recurrence using repeated measures of posttreatment PSA: A joint modeling approach. Biostatistics, 10(3), $535-549$.

Rizopoulos, D. (2010). JM: An R package for the joint modelling of longitudinal and time-to-event data. J. Stat. Softw., 35(9), 1-33.

Rizopoulos, D. (2012). Joint Models for Longitudinal and Time-To-Event Data: With Applications in R Boca Raton, Florida: CRC Press.

Rizopoulos, D. (2016). Package 'JM': Joint Modeling of Longitudinal and Survival Data. https:/cran.r-project.org/ web/packages/JM/JM.pdf, Accessed: 2016-09-30.

Rizopoulos, D. (2016). Package 'JMbayes': Joint Modeling of Longitudinal and Time-to-Event Data under a Bayesian Approach. Accessed: 2016-10-30.

Rizopoulos, D. (2016). The R package JMbayes for fitting joint models for longitudinal and time-to-event data using MCMC. J. Stat. Softw., 72(7), 1-46.

Rizopoulos, D. \& Ghosh, P. (2011). A Bayesian semiparametric multivariate joint model for multiple longitudinal outcomes and a time-to-event. Stat. Med., 30(12), 1366-1380.

Rizopoulos, D., Hatfield, L. A., Carlin, B. P. \& Takkenberg, J. J. M. (2014). Combining dynamic predictions from joint models for longitudinal and time-to-event data using Bayesian model averaging. J. Am. Stat. Assoc., 109(508), 1385-1397.

Rizopoulos, D., Verbeke, G. \& Lesaffre, E. (2009). Fully exponential Laplace approximations for the joint modelling of survival and longitudinal data. J. R. Stat. Soc. S. B., 71, 637-654.

Rondeau, V., Gonzalez, J. R., Mazroui, Y., Mauguen, A., Krol, A., Diakite, A., Laurent, A. \& Lopez, M. (2017). General Frailty Models: Shared, Joint and Nested Frailty Models (Package 'frailtypack'). https://cran.r-project.org/ web/packages/frailtypack/frailtypack.pdf, Online; accessed August 29, 2017.

Rondeau, V., Mazroui, Y. \& Gonzalez, J. R. (2012). Frailtypack: An R package for the analysis of correlated survival data with frailty models using penalized likelihood estimation or parametrical estimation. J. Stat. Softw., 47(4), 128.

Sudell, M. (2018). joineRmeta. https://cran.r-project.org/web/packages/joineRmeta/vignettes/joineRmeta.html, Accessed: 2018-04-05.

Sudell, M., Kolamunnage-Dona, R. \& Smith, C. T. (2018). joineRmeta: Joint Modelling for Meta-Analytic (MultiStudy) Data. https://cran.r-project.org/web/packages/joineRmeta/index.html, Accessed: 2018-04-05.

Sudell, M., Kolamunnage-Dona, R. \& Tudur-Smith, C. (2016). Joint models for longitudinal and time-to-event data: A review of reporting quality with a view to meta-analysis. BMC Med. Res. Methodol., 16(168), 1-11.

Taylor, J. M. G., Park, Y., Ankerst, D. P., Proust-Lima, C., Williams, S., Kestin, L., Bae, K., Pickles, T. \& Sandler, H. (2013). Real-time individual predictions of prostate cancer recurrence using joint models. Biometrics, 69, $206-213$.

Therneau, T. M. \& Lumley, T. (2016). Package 'survival': Survival Analysis. https://cran.r-project.org/web/packages/ survival/survival.pdf, Accessed: 2016-10-12.

Tseng, Y.-K., Hsieh, F. \& Wang, J.-L. (2005). Joint modelling of accelerated failure time and longitudinal data. Biometrika, 92(3), 587-603. 
Tsiatis, A. A. \& Davidian, M. (2004). Joint modeling of longitudinal and time-to-event data: An overview. Statistica Sinica, 14, 809-834.

Tsiatis, A. A., Degruttola, V. \& Wulfsohn, M. S. (1995). Modeling the relationship of survival to longitudinal data measured with error. Applications to survival and CD4 counts in patients with AIDS. J. Am. Stat. Assoc., 90(429), 27-37.

Wang, Y. \& Taylor, J. M. G. (2001). Jointly modeling longitudinal and event time data with application to acquired immunodeficiency syndrome. J. Am. Stat. Assoc., 96(455), 895-905.

Wang, W., Wang, W., Mosley, T. H. \& Griswold, M. E. (2017). A SAS macro for the joint modeling of longitudinal outcomes and multiple competing risk dropouts. Comput. Methods Prog. Biomed., 138, 23-30.

Williamson, P., Kolamunnage-Dona, R., Philipson, P. \& Marson, A. (2008). Joint modelling of longitudinal and competing risks data. Stat. Med., 27(30), 6426-6438.

Wulfsohn, M. S. \& Tsiatis, A. A. (1997). A joint model for survival and longitudinal data measured with error. Biometrics, 53(1), 330-339.

$\mathrm{Xu}$, C., Baines, P. D. \& Wang, J.-L. (2014). Standard error estimation using the EM algorithm for the joint modeling of survival and longitudinal data. Biostatistics, 15(4), 731-744.

Xu, C., Hadjipantelis, P. Z. \& Wang, J.-L. (2018). Semiparametric Joint Modeling of Survival and Longitudinal Data. https://cran.r-project.org/web/packages/JSM/JSM.pdf, Online; accessed February 11, 2019.

Yu, M., Law, N. J., Taylor, J. M. \& Sandler, H. M. (2004). Joint longitudinal-survival-cure models and their application to prostate cancer. Statistica Sinica, 14, 835-862.

Yuen, H. P. \& Mackinnon, A. (2016). Performance of joint modelling of time-to-event data with time-dependent predictors: An assessment based on transition to psychosis data. PeerJ, 4, 1-20.

Zhang, D., Chen, M.-H., Ibrahim, J. G., Boye, M. R. \& Shen, W. (2016). JMfit: A SAS macro for joint models of longitudinal and survival data. J. Stat. Softw., 71(3), 1-24.

Zhang, D., Chen, M.-H., Ibrahim, J. G., Boye, M. E., Wang, P. \& Shen, W. (2014). Assessing model fit in joint models of longitudinal and survival data with applications to cancer clinical trials. Stat. Med., 33(27), 4715-4733.

[Received April 2018, accepted February 2019] 